hep-ph/9406252

KEK-TH-376

KEK preprint 93-160

UT-659

March 1994

$\mathbf{H}$

\title{
Gluon and Charm Distributions in the Photon
}

\author{
K. Hagiwara, M. Tanaka and I. Watanabel \\ Theory Group, KEK, Tsukuba, Ibaraki 305, Japan \\ T. Izubuchi \\ Dept. of Physics, Univ. of Tokyo, Bunkyo-ku, Tokyo 113, Japan
}

(March 1994)

\begin{abstract}
We study systematically the sensitivity of the photon structure function data on the gluon contents of the photon, by using the leading order Altarelli-Parisi (AP) equations. Charm quark contribution is studied in the quark parton model and by using the massive quark AP equations of Glück, Hoffmann and Reya. The present photon structure function data are found to allow wide range of gluon distributions in the photon. We give a set of six scale-dependent parton distributions in the photon (WHIT1 to WHIT6), which have systematically different gluon contents. Sensitivity of the structure function at small $x$ and that of the total charm quark production cross section to the effective
\end{abstract}


gluon distribution are discussed.

12.38.-t

Typeset using REVTEX 


\section{INTRODUCTION}

The deep structure of the photon has been expected to be calculable perturbatively in QCD [1], except at small $x$ [2]. In practice, however, non-perturbative effects are found to be significant [3, [4] at present experiments, where the electromagnetic structure of WeizsäckerWilliams quasi-real photon [5] has been measured up to the momentum transfer scale $Q^{2}$ $=100 \mathrm{GeV}^{2}$ in $e^{+} e^{-}$collision experiments. Several parametrizations of the scale-dependent effective parton distributions in the photon have been proposed; some are based on plausible dynamical assumptions [3,6] 9] and the others [4, 10] have been obtained by fitting phenomenologically to the photon structure function data [11 17]. These parametrizations typically have similar quark distributions which are directly constrained by the structure function data. On the other hand, wildly different gluon distributions have been proposed since the electromagnetic structure of the photon is rather insensitive to its gluon content.

Recently TRISTAN experiments [18,19] have shown evidence for the effective gluon content of the photon in two-photon production of high transverse momentum $\left(p_{T}\right)$ jets. The observed jet production cannot be understood without the contribution from gluons in the colliding photons, whereas it does not allow a very hard gluon distribution [10] that is consistent with the structure function data.

More recently, the TRISTAN experiments have reported evidence for copious production of charmed particles in the two-photon collision process, in their various decay modes and at various $p_{T}$ range [20 22]. The charm production rate has been found to be particularly sensitive to the gluon distribution in the photon [23] and that the present data tend to prefer those parametrizations with large gluon content at small $x(x \lesssim 0.1)$.

The recent data on the jet and charm production in the two-photon process thus give us evidence and some quantitative information of the gluon content of the photon, but they are not yet accurate enough to determine directly the effective gluon distribution. It is therefore

desirable to have a set of effective parton distributions in the photon with systematically different gluon distributions, so that we can learn more about the photon structure from 
these experiments.

In this paper we study all the available photon structure function data [1] 17, 24 26] at $4 \mathrm{GeV}^{2} \leq Q^{2} \leq 100 \mathrm{GeV}^{2}$ in the leading order of perturbative QCD and, find a new set of the effective scale-dependent parton distributions in the photon, named WHIT1 to WHIT6, which are all consistent with the present data of the photon structure function and have systematically different gluon contents. We study carefully the charm quark contributions to the observed structure functions, which are evaluated by using the lowest order quark parton model matrix elements $\left(\gamma^{*} \gamma \rightarrow c \bar{c}\right.$ and $\left.\gamma^{*} g \rightarrow c \bar{c}\right)$ and the massive Altarelli-Parisi (AP) equations [27]. We find that the photon structure function has a sensitivity to the gluon distribution at small $x$, but that a careful analysis is needed to determine experimentally the photon structure in this region. Predictions are also given for the total charm quark pair production cross section in the two-photon collision process at $e^{+} e^{-}$colliders.

We note here that the next-to-leading order correction to the massless inhomogeneous AP equations has been known for a while [3, 28, 30], and some phenomenological analyses [6] were performed at this level. Recently, the complete next-to-leading order correction has been obtained for the massive quark production process [31]. We work in the leading order of QCD, nevertheless, since errors in the experimental data as well as the theoretical uncertainties associated with the gluon contents of the photon are so large that the leading order approach is more suited to discuss them systematically.

The paper is organized as follows. In Sec. II, we review the AP equations that govern the effective parton distributions in the photon and introduce the notion of 'valence' and 'sea' components of the quark distributions. We discuss our parametrizations of the initial quark and gluon distributions and study charm contributions to the structure function. In Sec. III, we introduce all available photon structure function data, give a criterion to remove several low $x$ experimental data, and then make a global fit of the initial light-quark distribution functions by using the leading order inhomogeneous AP equations. The fit is repeated by systematically changing the magnitude and the shape of the initial gluon distribution. The minimal $\chi^{2}$ of the fit as well as the distribution of the deviation of each data point 
from the best fit curve is examined carefully. Six effective parton distributions which have systematically different initial gluon distributions, WHIT1 to WHIT6, are then introduced and examined. In Sec. IV, we discuss effective heavy quark distributions in the photon by using the quark parton model (QPM) and the massive AP equations. In Sec. $\nabla$, predictions are given for the total charmed particle production cross section in the two-photon process at $e^{+} e^{-}$colliders. Sec. V1 summarizes our findings. The details on the numerical methods that we use in order to solve the AP equation with and without charm quark mass effects are give in Appendix A, and the parametrizations of our effective parton distributions in the photon are described in Appendix B.

\section{MODEL}

In this section we explain the framework adopted in this work to calculate the effective parton distribution functions in the photon and the photon structure function $F_{2}^{\gamma}\left(x, Q^{2}\right)$.

\section{A. Inhomogeneous Altarelli-Parisi equations}

In the $Q^{2}$ and $x$ region where the lightest $n_{f}$-flavor quarks can be produced, we have $n_{f}$ quark distributions and $n_{f}$ anti-quark distributions in addition to the gluon distribution in the photon. These are denoted by $q_{i}\left(x, Q^{2}\right), \bar{q}_{i}\left(x, Q^{2}\right)\left(i=1\right.$ to $\left.n_{f}\right)$, and $g\left(x, Q^{2}\right)$ respectively. Apparently, the relation $q_{i}\left(x, Q^{2}\right)=\bar{q}_{i}\left(x, Q^{2}\right)$ holds for each flavor.

The $Q^{2}$ evolution of these parton distributions is described by the inhomogeneous Altarelli-Parisi (AP) equations in the leading logarithmic approximation [32. For massless $n_{f}$-flavor case the AP equations can be written as follows:

$$
\begin{aligned}
& \frac{d q_{i}\left(x, Q^{2}\right)}{d t}=\frac{\alpha}{2 \pi} e_{i}^{2} P_{q \gamma}(x)+\frac{\alpha_{s}\left(Q^{2}\right)}{2 \pi}\left[P_{q q}(x) \otimes q_{i}\left(x, Q^{2}\right)+P_{q g}(x) \otimes g\left(x, Q^{2}\right)\right], \\
& \frac{d g\left(x, Q^{2}\right)}{d t}=\frac{\alpha_{s}\left(Q^{2}\right)}{2 \pi}\left[2 P_{g q}(x) \otimes \sum_{i=1}^{n_{f}} q_{i}\left(x, Q^{2}\right)+P_{g g}\left(x, n_{f}\right) \otimes g\left(x, Q^{2}\right)\right]
\end{aligned}
$$

where $i=1$ to $n_{f}, t=\log Q^{2} / \Lambda^{2}$ with $\Lambda$ being the QCD scale, and $P_{i j}$ 's are the parton 
splitting functions [33] whose explicit forms are given in Eq. (A4) of Appendix A. The convolution integral is defined as $a(x) \otimes b(x)=\int_{x}^{1} d y / y a(x / y) b(y)$.

As we show in the latter sections, the charm quark contribution to the photon structure function can be most conveniently calculated from the lowest order quark parton model processes $\left(\gamma^{*} \gamma \rightarrow c \bar{c}\right.$ and $\left.\gamma^{*} g \rightarrow c \bar{c}\right)$ at present energies $\left(Q^{2} \lesssim 100 \mathrm{GeV}^{2}\right)$, by excluding the charm quark distribution in the photon. Only at higher $Q^{2}$ we introduce the effective charm quark distribution that evolves according to the massive $n_{f}=4$ AP equations of Ref. [27]. At very high $Q^{2}$, we may neglect the charm quark mass and employ the massless inhomogeneous AP equations of Eq. (2.1) with $n_{f}=4$. The matching of the quark parton model description with the effective heavy quark distribution in the massive AP equations is discussed in Sec. IV.

Hence in the analysis of the present structure function data that probe the photon structure up to $Q^{2} \sim 100 \mathrm{GeV}^{2}$, we introduce only three light-quark distributions $\left(n_{f}=3\right)$. Furthermore, in order to find a plausible parametrization of these quark distributions for the fit, we find it convenient to introduce the notion of 'valence' and 'sea' quark distributions. The 'valence' quarks are produced by the photon and the 'sea' quarks originate from the gluons. According to these definitions, the valence and sea quark distributions are written in terms of the original quark distributions $q_{i}$ 's:

$$
\begin{gathered}
q_{v}\left(x, Q^{2}\right)=2 \sum_{i=1}^{3} \frac{e_{i}^{2}\left\langle e^{2}\right\rangle-\left\langle e^{2}\right\rangle^{2}}{\left\langle e^{4}\right\rangle-\left\langle e^{2}\right\rangle^{2}} q_{i}\left(x, Q^{2}\right), \\
q_{\text {sea }}\left(x, Q^{2}\right)=2 \sum_{i=1}^{3} \frac{\left\langle e^{4}\right\rangle-e_{i}^{2}\left\langle e^{2}\right\rangle}{\left\langle e^{4}\right\rangle-\left\langle e^{2}\right\rangle^{2}} q_{i}\left(x, Q^{2}\right),
\end{gathered}
$$

where $\left\langle e^{2}\right\rangle=2 / 9$ and $\left\langle e^{4}\right\rangle=2 / 27$ for $n_{f}=3$. Note that the singlet and non-singlet quark distributions, $q_{S}\left(x, Q^{2}\right)$ and $q_{N S}\left(x, Q^{2}\right)$, respectively, are related to our valence and sea quark distributions by

$$
\begin{aligned}
q_{S}\left(x, Q^{2}\right) & \equiv 2 \sum_{i=1}^{3} q_{i}\left(x, Q^{2}\right), \\
& =q_{v}\left(x, Q^{2}\right)+q_{s e a}\left(x, Q^{2}\right), \\
q_{N S}\left(x, Q^{2}\right) & \equiv 2 \sum_{i=1}^{3}\left[e_{i}^{2}-\left\langle e^{2}\right\rangle\right] q_{i}\left(x, Q^{2}\right),
\end{aligned}
$$




$$
=\left[\frac{\left\langle e^{4}\right\rangle}{\left\langle e^{2}\right\rangle}-\left\langle e^{2}\right\rangle\right] q_{v}\left(x, Q^{2}\right) .
$$

The photon structure function $F_{2}^{\gamma}\left(x, Q^{2}\right)$ can be written in terms of $q_{v}\left(x, Q^{2}\right)$ and $q_{\text {sea }}\left(x, Q^{2}\right)$ as

$$
\begin{aligned}
F_{2}^{\gamma}\left(x, Q^{2}\right) & \equiv 2 x \sum_{i=1}^{n_{f}} e_{i}^{2} q_{i}\left(x, Q^{2}\right) \\
& =x\left[\frac{\left\langle e^{4}\right\rangle}{\left\langle e^{2}\right\rangle} q_{v}\left(x, Q^{2}\right)+\left\langle e^{2}\right\rangle q_{\text {sea }}\left(x, Q^{2}\right)\right]+\text { heavy quarks } .
\end{aligned}
$$

Heavy quark contributions will be discussed in subsection ПТ. When we neglect small mass differences among the light three flavors, we can express the $u, d$ and $s$ distributions in terms of $q_{v}$ and $q_{\text {sea }}$ :

$$
\begin{array}{r}
u\left(x, Q^{2}\right)=\frac{1}{3} q_{v}\left(x, Q^{2}\right)+\frac{1}{6} q_{\text {sea }}\left(x, Q^{2}\right), \\
d\left(x, Q^{2}\right)=s\left(x, Q^{2}\right)=\frac{1}{12} q_{v}\left(x, Q^{2}\right)+\frac{1}{6} q_{\text {sea }}\left(x, Q^{2}\right) .
\end{array}
$$

The AP equations of Eq. (2.1) with $n_{f}=3$ can be rewritten in terms of the valence-quark, the sea-quark and the gluon distributions:

$$
\begin{aligned}
\frac{d q_{v}\left(x, Q^{2}\right)}{d t} & =\frac{\alpha}{2 \pi}\left\langle e^{2}\right\rangle P_{q \gamma}(x)+\frac{\alpha_{s}\left(Q^{2}\right)}{2 \pi} P_{q q}(x) \otimes q_{v}\left(x, Q^{2}\right), \\
\frac{d q_{s e a}\left(x, Q^{2}\right)}{d t} & =\frac{\alpha_{s}\left(Q^{2}\right)}{2 \pi}\left[2 \cdot 3 P_{q g}(x) \otimes g\left(x, Q^{2}\right)+P_{q q}(x) \otimes q_{s e a}\left(x, Q^{2}\right)\right], \\
\frac{d g\left(x, Q^{2}\right)}{d t} & =\frac{\alpha_{s}\left(Q^{2}\right)}{2 \pi}\left[P_{g q}(x) \otimes\left\{q_{v}\left(x, Q^{2}\right)+q_{\text {sea }}\left(x, Q^{2}\right)\right\}+P_{g g}(x, 3) \otimes g\left(x, Q^{2}\right)\right] .
\end{aligned}
$$

It is now clearly seen that the valence-quarks are produced by the photon, the sea-quarks are produced by the gluon, while the gluon is produced by the valence-quarks, the sea-quarks and the gluon itself. Once a set of initial parton distributions at $Q^{2}=Q_{0}^{2}$ is given, we can predict the parton distributions at any $Q^{2}\left(>Q_{0}^{2}\right)$ by solving the above equations numerically. The numerical methods which we use to solve these equations are explained in Appendix A.

\section{B. Initial parton distributions}

To solve the AP equations of Eq. (2.6), we have to specify a set of initial parton distributions at $Q^{2}=Q_{0}^{2}$. All the non-perturbative features of the photon structure are included 
in these initial conditions. We use $Q_{0}^{2}=4 \mathrm{GeV}^{2}$ throughout our analysis in order that our perturbation approximation works well.

As an initial valence-quark distribution, we take the following functional form:

$$
x q_{v}\left(x, Q_{0}^{2}\right) / \alpha=A_{v} x^{B_{v}}(1-x)^{C_{v}} / B\left(B_{v}+1, C_{v}+1\right),
$$

where $A_{v}, B_{v}$ and $C_{v}$ are the free parameters which will be fitted to the experimental data, and $B(\alpha, \beta)$ is the beta function that ensures the normalization,

$$
\left\langle x q_{v}\left(x, Q_{0}^{2}\right)\right\rangle / \alpha \equiv \int_{0}^{1} d x x q_{v}\left(x, Q_{0}^{2}\right) / \alpha=A_{v}
$$

for the energy fraction $\left\langle x q_{v}\left(x, Q_{0}^{2}\right)\right\rangle$ of the valence-quarks in the photon.

As for the initial gluon distribution, we adopt the simple form

$$
x g\left(x, Q_{0}^{2}\right) / \alpha=A_{g}\left(C_{g}+1\right)(1-x)^{C_{g}},
$$

with two parameters, $A_{g}$ and $C_{g}$. Again the normalization factor is chosen such that

$$
\left\langle x g\left(x, Q_{0}^{2}\right)\right\rangle / \alpha=\int_{0}^{1} d x x g\left(x, Q_{0}^{2}\right) / \alpha=A_{g}
$$

The present structure function data are not accurate enough to determine the gluon parameters $A_{g}$ and $C_{g}$. We therefore perform fit by the valence-quark parameters, $A_{v}, B_{v}$ and $C_{v}$, by varying systematically the normalization $\left(A_{g}\right)$ and the shape $\left(C_{g}\right)$ of the initial gluon distribution.

Before starting the fit to the data, we discuss plausible range of the gluon distribution parameters that we should explore. We obtain constraints on the ratio of the gluon energy fraction $\left(A_{g}\right)$ to the valence-quark energy fraction $\left(A_{v}\right)$ as follows [7]. At sufficiently low momentum transfer scale $\left(Q^{2} \lesssim 0.5 \mathrm{GeV}^{2}\right)$, only the long wavelength components of the photon are probed and the quark-antiquark pair produced from the photon undergoes nonperturbative soft QCD dynamics that resembles the one which makes the quark-antiquark pair form the low-lying vector boson. The photon structure is then expected to have components similar to those of the vector bosons, in particular the $\rho$ meson that couples strongly to 
the photon. Although we do not know the structure of $\rho$, we expect the soft QCD dynamics to be insensitive to the total spin of the system and that it may be similar to the observed $\pi$ structure [34]. If the photon had only this soft component, its deep structure should also be similar and we expect

$$
\frac{\left\langle x g\left(x, Q_{0}^{2}\right)\right\rangle_{{ }^{\prime} \rho}{ }^{\prime}}{\left\langle x q_{v}\left(x, Q_{0}^{2}\right)\right\rangle_{{ }^{\prime} \rho}} \sim \frac{\left\langle x g\left(x, Q_{0}^{2}\right)\right\rangle_{\pi}}{\left\langle x q_{v}\left(x, Q_{0}^{2}\right)\right\rangle_{\pi}} \sim 1 .
$$

In fact this ratio is common in the nucleon structure as well [35,27 and we can regard this ratio as an universal one from soft QCD dynamics.

The photon, however, differs from the vector boson in that it is a source of a quarkpair with an arbitrary short wave-length. As the momentum transfer scale grows $\left(Q^{2} \gtrsim\right.$ $0.5 \mathrm{GeV}^{2}$ ), one is more and more sensitive to these short wave-length components which dominate the photon structure at asymptotically high $Q^{2}$. Although the transition from the regime where the vector meson-like component dominates to the regime where the short wave-length component dominates is gradual and it is governed by the non-perturbative dynamics, we may infer the effect of the latter component from its asymptotic behavior that can be calculated perturbatively. In particular, for the ratio of the gluon to the valence-quark energy fraction, we expect

$$
\left.\frac{\left\langle x g\left(x, Q^{2}\right)\right\rangle}{\left\langle x q_{v}\left(x, Q^{2}\right)\right\rangle}\right|_{Q^{2} \rightarrow \infty}=\frac{3616}{10611} \simeq \frac{1}{3},
$$

for three light quark flavors $\left(n_{f}=3\right)$. At the momentum transfer scale $Q_{0}^{2}=4 \mathrm{GeV}^{2}$, it is hence natural to expect the ratio to lie somewhere between the two extremes Eq.(2.11) and (2.12):

$$
\frac{1}{3} \lesssim \frac{\left\langle x g\left(x, Q_{0}^{2}\right)\right\rangle}{\left\langle x q_{v}\left(x, Q_{0}^{2}\right)\right\rangle}=\frac{A_{g}}{A_{v}} \lesssim 1
$$

We shall see in the next section that the valence-quark fraction $A_{v}$ is determined to be about unity by the experimental data of $F_{2}^{\gamma}\left(x, Q^{2}\right)$. We will hence examine the parameter range $1 / 3 \lesssim A_{g} \lesssim 1$ for the gluon energy fraction.

Finally, we note that the sea-quark distribution is intimately related to the gluon distribution and that one cannot choose them independently. Although the sea-quark distribution 
is in principle observable from the small $x$ behavior of the photon structure function, we find that the present experimental determination of the small $x$ structure of the photon suffers from an uncertainty associated with the unfolding technique adopted by most experiments [36]: this will be discussed in Sec. [II]. We therefore estimate the input sea-quark distribution by using the quark parton model cross section for the process $\gamma^{*} g \rightarrow q \bar{q}$ :

$$
x q_{\text {sea }}\left(x, Q_{0}^{2}\right)=3 \frac{\alpha_{s}\left(Q_{0}^{2}\right)}{2 \pi} \int_{a x}^{1} d y w\left(\frac{x}{y}, \frac{m^{2}}{Q_{0}^{2}}\right) g\left(y, Q_{0}^{2}\right),
$$

where $a=1+4 m^{2} / Q_{0}^{2}$ and

$$
\begin{aligned}
w(z, r)=z[\beta\{-1+8 z(1-z)-4 r z(1-z)\} & \\
& \left.+\left\{z^{2}+(1-z)^{2}+4 r z(1-3 z)-8 r^{2} z^{2}\right\} \log \frac{1+\beta}{1-\beta}\right]
\end{aligned}
$$

with $\beta=\sqrt{1-4 r z /(1-z)}$. The sea-quark mass $m$, which is taken to be common for the three light flavors, $m_{u}=m_{d}=m_{s}=m$, plays the role of the cut-off and we choose it to be $0.5 \mathrm{GeV}$. Here and throughout our analysis we adopt the leading order form of the QCD running coupling constant

$$
\frac{\pi}{\alpha_{s}\left(Q^{2}\right)}=\frac{25}{12} \ln \frac{Q^{2}}{\Lambda_{4}^{2}}-\frac{1}{6} \ln \frac{Q^{2}}{4 m_{b}^{2}} \Theta\left(Q^{2}-4 m_{b}^{2}\right),
$$

with $\Lambda_{4}=0.4 \mathrm{GeV}$ and $m_{b}=5 \mathrm{GeV}$. Note that the effective number of quark flavors that governs the running of the coupling constant is chosen independently of the number $n_{f}$ of massless quark flavors in the AP equations of Eq. (2.1). An accurate prescription for the choice of the effective number of flavors is found only in the next-to-leading order level [31].

We remark here that the above prescription leads naturally to a larger sea-quark input as the gluon input is enhanced. In particular, we find for the energy fraction ratio that

$$
\frac{\left\langle x q_{\text {sea }}\left(x, Q_{0}^{2}\right)\right\rangle}{\left\langle x g\left(x, Q_{0}^{2}\right)\right\rangle} \sim 0.12
$$

holds almost independently of the input gluon parameters $A_{g}$ and $C_{g}$ in the region which we will discuss. The ratio increases with decreasing light-quark mass, and reaches 0.2 at $m \sim 0.3 \mathrm{GeV}$. The ratio is about 0.3 in the parametrization of the $\pi$ structure [34, and its 
perturbative asymptotic value is calculated to be 0.16 for $n_{f}=3$. Our sea-quark input is hence rather conservative for a given input gluon distribution.

\section{Charm contribution}

The charm quark cannot be incorporated into the massless AP equations in the region of moderate $Q^{2}$, say $Q^{2} \leq 100 \mathrm{GeV}^{2}$, which has so far been probed by experiments. We should take into account the quark mass effect by using the massive-quark AP equation of M. Glück, E. Hoffmann, and E. Reya [27], and more accurately by incorporating the full next-to-leading order corrections [31]. We find by comparing with the results of the leading order massivequark AP equations that the charm quark contribution to the photon structure function is well approximated by the sum of the contributions from the quark parton model processes $\gamma^{*} \gamma \rightarrow c \bar{c}$ and $\gamma^{*} g \rightarrow c \bar{c}$ at $Q^{2} \leq 100 \mathrm{GeV}^{2}$. Beyond $Q^{2} \sim 100 \mathrm{GeV}^{2}$ the radiation of gluons off charm quarks is no longer negligible and, we should solve the massive-quark inhomogeneous AP equations. At large enough $Q^{2}$, the charm quark mass effect to the $Q^{2}$-evolution would become negligible and we can use the massless AP equations of Eq. (2.1) with $n_{f}=4$. The matching of the distributions should then be made at appropriately large $Q^{2}$. We will find that the charm-quark mass effects are not negligible even at $Q^{2} \sim 100 \mathrm{GeV}^{2}$. With the same criterion, the bottom quark contribution can be estimated by the lowest order process $\gamma^{*} \gamma \rightarrow b \bar{b}$ and $\gamma^{*} g \rightarrow b \bar{b}$ up to about $Q^{2} \sim 1000 \mathrm{GeV}^{2}$, above which we may introduce the effective b-quark distribution that follows the massive $n_{f}=5 \mathrm{AP}$ equations. More accurate quantitative treatment [31] will become useful in the future when both the quark and gluon distributions are measured accurately from experiments.

The charm-quark contributions to the photon structure function are thus calculated by the quark parton model at $Q^{2}<100 \mathrm{GeV}^{2}$. The contribution of the direct process $\left(\gamma^{*} \gamma \rightarrow c \bar{c}\right)$ is given by

$$
\left.F_{2, c}^{\gamma}\left(x, Q^{2}\right)\right|_{\text {direct }}=3 \frac{\alpha}{\pi} e_{c}^{4} w\left(x, \frac{m_{c}^{2}}{Q^{2}}\right)
$$


where $e_{c}=2 / 3$ is the charm-quark electric charge and the function $w(x, r)$ is given in Eq. (2.15). In our numerical analysis, we take $m_{c}=1.5 \mathrm{GeV}$. For the resolved process $\left(\gamma^{*} g \rightarrow c \bar{c}\right)$, we have

$$
\left.F_{2, c}^{\gamma}\left(x, Q^{2}\right)\right|_{\text {resolved }}=\frac{\alpha_{s}\left(Q^{2}\right)}{2 \pi} e_{c}^{2} \int_{a x}^{1} d y w\left(\frac{x}{y}, \frac{m_{c}^{2}}{Q^{2}}\right) g\left(y, Q^{2}\right),
$$

where $a=1+4 m_{c}^{2} / Q^{2}$, and the gluon distribution $g\left(x, Q^{2}\right)$ is given by solving the massless $n_{f}=3$ AP equations of Eq. (2.6) with the initial parton distributions of Eqs. (2.7), (2.9) and 2.14$)$.

The validity of our simple quark parton model calculation depends on how much the gluon emission by the charm quark distorts the effective charm quark distribution in the photon. The magnitude of this effect can be studied by using the massive AP equations for the charm quark and is presented in Sec. IV.

\section{FIT TO THE DATA}

\section{A. Data}

In order to find good initial parton distributions at the energy scale $Q_{0}^{2}=4 \mathrm{GeV}^{2}$, we refer to all the available experimental data of the photon structure function at $Q^{2}>Q_{0}^{2}$. In our analysis we use the data obtained by 8 groups at PETRA, PEP, TRISTAN and LEP $e^{+} e^{-}$colliders which are listed in Table $\mathbb{1}$.

We note here that not all the experimental data points are taken into account in our fit. First, we do not use the data at $\left\langle Q^{2}\right\rangle$ lower than $4.0 \mathrm{GeV}^{2}$. Second, we accept only those data points at small $x$ where the following inequality holds:

$$
x_{\text {lower edge of the bin }}>\frac{\left\langle Q^{2}\right\rangle}{\left\langle Q^{2}\right\rangle+\left(W_{\text {vis }}^{\max }\right)^{2}} \text {. }
$$

Here $W_{\text {vis }}^{\max }$ is the experimental cut on the visible invariant mass of the final hadron system. Since those data points that violate the condition Eq.(3.1) are obtained at near the boundary of the experimental acceptance and since the sea-quark contribution to the structure function 
can be rather singular at the low $x$ region, they may suffer from large systematic uncertainties in the unfolding procedure [36]. As a result of the above two requirements, 47 data points

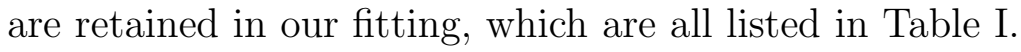

\section{B. Fit}

By fitting our theoretical predictions for the photon structure function to these experimental data, we tune the parameters of the initial valence-quark distribution $A_{v}, B_{v}$ and $C_{v}$. We repeat the fit by varying the initial gluon distribution parameters $A_{g}$ and $C_{g}$ systematically, while keeping the strong coupling constant and the charm quark mass fixed at $\Lambda_{4}=0.4 \mathrm{GeV}$ and $m_{c}=1.5 \mathrm{GeV}$, respectively. In particular, we examine the case with $A_{g}$ $=0.5,1$ and 1.5 systematically by changing $C_{g}$ and find little sensitivity of the structure function data to the shape parameter $C_{g}$. The fit results for arbitrarily chosen 12 cases are summarized in Table [1]. Although there is a tendency in the data that prefers small $A_{g}$ (small gluonic energy fraction) and small $C_{g}$ (hard gluon distribution), it is caused by a few data points at small $x$ with relatively large deviations from the best fit curve, as we will discuss below. We choose three representative $C_{g}$ values $\left(C_{g}=3,9,15\right)$ for each of the normalizations $A_{g}=0.5$ and 1 that are consistent with the ansatz Eq. (2.13). These gluon inputs are named as WHIT1 to WHIT6, respectively, as shown in Table III.

Fig 1 illustrates the matching of the data and the theoretical curves, and Fig. 2 shows the distribution of the deviation of each data point from the best fit value, $\left(F_{2}(x)_{\mathrm{fit}}-\right.$ $\left.F_{2}(x)_{\text {data }}\right) / \sigma\left(F_{2}(x)_{\text {data }}\right)$. As can be seen from Fig. 1 and Fig. 2, all of WHIT1 to WHIT6 gluon distributions give similar quality of fits to the photon structure function data. The mild dependence of the best fit $\chi^{2}$ value on the choice of the initial gluon distribution parameters

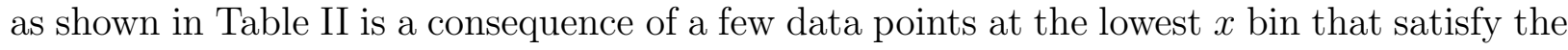
criterion Eq. (3.1): see Fig. 2. In Fig. 2, deviations of those data points that are removed from the fit by the criterion Eq. (3.1) are also indicated by dashed lines. The large $A_{g}$ large $C_{g}$ gluon distributions lead to a significant rise in the structure function at small $x$, and 
a naive integration of the structure function in the given $x$-bin tends to be very sensitive to the lower edge of the lowest $x$-bin as is seen in Fig. 1. After imposing the selection criterion Eq. (3.1), such sensitivity to the very low $x$ behavior of the structure function is almost completely lost, as can be seen from the deviations of the data points that are connected by the solid lines in Fig. 2. In view of the relatively large theoretical uncertainty in simulating hadronic events at small $x$, we conclude that the present experimental data on the photon structure function have poor sensitivity to the gluonic content of the photon. The normalization of the valence-quark distribution $A_{v}$ is found to be roughly 1 , regardless of the difference in the sea-quark contribution that depends strongly on our gluon inputs.

We find from Table [1] that the best fit values of the initial valence-quark parameters are almost the same for different $C_{g}$ 's for a common $A_{g}$. Hence we introduce a 'standard' set of the valence-quark parameters for each $A_{g}$; i.e.

$$
\begin{aligned}
& A_{v}=0.94, \quad B_{v}=0.50, \quad C_{v}=0.25 \quad \text { for } A_{g}=0.5, \\
& A_{v}=0.89, \quad B_{v}=0.70, \quad C_{v}=0.45 \quad \text { for } A_{g}=1.0 .
\end{aligned}
$$

We calculate the $\chi^{2}$ values for various $C_{g}$ 's by fixing the normalization $A_{g}$ and the associated valence-quark inputs as above, and the result is summarized in Table III. The $C_{g}$ dependence

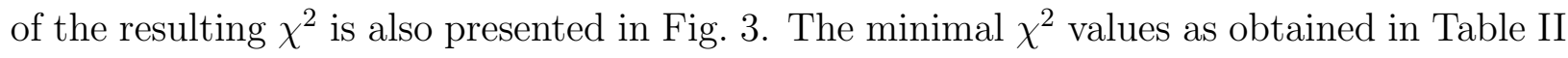
by tuning the valence quark parameters are also shown by large symbols. The tuning of the valence-quark parameters do not improve the fit much. We therefore adopt the common valence-quark input of Eq. (3.2a) for the sets WHIT1 to WHIT3, while that of Eq.(3.2b) for the sets WHIT4 to WHIT6, and hereafter we call the parton distributions with these inputs as WHIT1 to WHIT6. The slightly small valence-quark contributions of Eq.(3.2b) at small $x$ partially compensates for the larger sea-quark contributions associated with the large gluon inputs of WHIT4 to WHIT6. 


\section{Gluon distribution}

As we described above, we present a set of six effective parton distributions in the photon with systematically different gluon contents. We show in Fig. 囵 all the gluon distributions (WHIT1 to WHIT6) at three momentum transfer scales, $Q^{2}=4,20$ and $100 \mathrm{GeV}^{2}$. The

area under the curves at $Q^{2}=4 \mathrm{GeV}^{2}$ is given by the normalization $A_{g}=0.5$ for WHIT1 to WHIT3, and $A_{g}=1.0$ for WHIT4 to WHIT6. The shape of the distribution becomes softer as we move from WHIT1 to 3, and from WHIT4 to 6 in each set. The huge difference in the initial gluon distributions tends to diminish at higher $Q^{2}$, as expected from the asymptotic behavior of the solution of the inhomogeneous AP equations.

Also shown in Fig. \ for comparison are the effective gluon distributions of GRV [9], DG [4] and LAC1 [10] at the three momentum transfer scales. We note that in the $x$ and $Q^{2}$ range as shown in Fig. \&, our WHIT1 effective gluon distribution is similar to the gluon distribution of GRV [9], while WHIT6 gluon distribution behaves similarly to that of LAC1 [10]. It should be noted, however, that LAC1 parametrization for the effective gluon distribution is more singular at $x \rightarrow 0$ than that of WHIT6, which results in a very large energy fraction $\left\langle x g\left(x, Q_{0}^{2}\right)\right\rangle / \alpha=2.37$ at $Q_{0}^{2}=4 \mathrm{GeV}^{2}$, in conflict with our ansatz Eq. (2.13) with $A_{v} \sim 1$. Accurate measurements of the photon structure functions at very small $x$ as well as the high energy behavior of the charm and mini-jet production cross section at $\gamma \gamma$ and $\gamma p$ collisions will be able to distinguish the different small $x$ behavior of these two effective gluon distributions.

\section{EFFECTIVE HEAVY-QUARK DISTRIBUTIONS IN THE PHOTON}

In this section, we compare the result of the quark parton model (QPM) calculation of the effective charm quark distribution with that of the massive inhomogeneous AltarelliParisi(AP) equations [27]. We expect that the QPM approach is appropriate at low momentum transfer scale where the charm quark mass effect is significant. At high momentum 
transfer $Q^{2} / m_{c}^{2} \gg 1$, gluon emission from charm quarks is no longer negligible and we need the massive-quark AP equations to sum up the leading effects. On the other hand, the approach of [27] neglects the charm quark mass effects in gluon emission from charm quarks, and hence it may overestimate the gluon emission effects at low $Q^{2}$. We therefore use the QPM prescription for the effective charm quark distribution at $Q^{2} \leq 100 \mathrm{GeV}^{2}$ and switch to the solution of the massive quark $\mathrm{AP}$ equations at $Q^{2} \geq 100 \mathrm{GeV}^{2}$.

The QPM charm quark distribution consists of the valence part and the sea part and is defined as

$$
c^{\mathrm{QPM}}\left(x, Q^{2}\right)=c_{v}^{\mathrm{QPM}}\left(x, Q^{2}\right)+c_{\text {sea }}^{\mathrm{QPM}}\left(x, Q^{2}\right),
$$

where

$$
\begin{aligned}
& c_{v}^{\mathrm{QPM}}\left(x, Q^{2}\right)=\left.\frac{1}{2 x e_{c}^{2}} F_{2, c}^{\gamma}\left(x, Q^{2}\right)\right|_{\text {direct }}, \\
& c_{\text {sea }}^{\mathrm{QPM}}\left(x, Q^{2}\right)=\left.\frac{1}{2 x e_{c}^{2}} F_{2, c}^{\gamma}\left(x, Q^{2}\right)\right|_{\text {resolved }},
\end{aligned}
$$

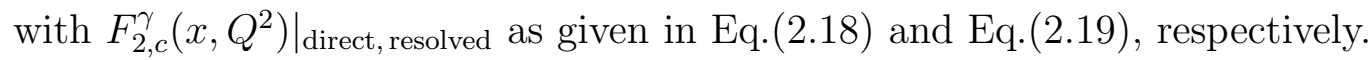

As is clear from these definitions, the effective charm quark distribution calculated by the QPM reproduces the photon structure function well, but we should expect large processdependent threshold corrections when it is used for other processes with an equivalent real charm quark in the photon.

At high $Q^{2}$, we expect that emission of collinear gluons from charm quarks becomes significant and we solve the massive-quark inhomogeneous AP equations

$$
\begin{aligned}
& \frac{d q_{i}\left(x, Q^{2}\right)}{d t}=\frac{\alpha}{2 \pi} e_{i}^{2} P_{q \gamma}(x)+\frac{\alpha_{s}\left(Q^{2}\right)}{2 \pi}\left[P_{q q}(x) \otimes q_{i}\left(x, Q^{2}\right)+P_{q g}(x) \otimes g\left(x, Q^{2}\right)\right], \\
& \frac{d g\left(x, Q^{2}\right)}{d t}=\frac{\alpha_{s}\left(Q^{2}\right)}{2 \pi}\left[P_{g q}(x) \otimes 2\left(\sum_{i} q_{i}\left(x, Q^{2}\right)+c\left(x, Q^{2}\right)\right)+P_{g g}(x, 4) \otimes g\left(x, Q^{2}\right)\right], \\
& \frac{d c\left(x, Q^{2}\right)}{d t}=\frac{\alpha}{2 \pi} e_{c}^{2} P_{c \gamma}\left(x, Q^{2}\right)+\frac{\alpha_{s}\left(Q^{2}\right)}{2 \pi}\left[P_{q q}(x) \otimes c\left(x, Q^{2}\right)+P_{c g}\left(x, Q^{2}\right) \otimes g\left(x, Q^{2}\right)\right],
\end{aligned}
$$

where $i=u, d, s$, and $P_{c \gamma}$ and $P_{c g}$ are the photon and gluon to massive-quark splitting functions, respectively, as defined by [27] 


$$
P_{c g}\left(x, Q^{2}\right)=\frac{1}{2} \theta(1 / a-x) \frac{d}{d t} \frac{w\left(x, m_{c}^{2} / Q^{2}\right)}{x}, \quad P_{c \gamma}\left(x, Q^{2}\right)=6 P_{c g}\left(x, Q^{2}\right),
$$

with $a=1+4 m_{c}^{2} / Q^{2}$. The function $w(x, r)$ is defined in Eq. (2.15).

As in the case of light quarks, it is convenient to divide the charm-quark distribution into the valence and the sea parts:

$$
c\left(x, Q^{2}\right)=c_{v}\left(x, Q^{2}\right)+c_{s e a}\left(x, Q^{2}\right) .
$$

The valence-charm-quark comes from the photon and the sea-charm-quark comes from the gluon. Eq.(4.3d) can then be split into the following two equations for $c_{v}$ and $c_{\text {sea }}$ :

$$
\begin{aligned}
\frac{d c_{v}\left(x, Q^{2}\right)}{d t} & =\frac{\alpha}{2 \pi} e_{c}^{2} P_{c \gamma}\left(x, Q^{2}\right)+\frac{\alpha_{s}\left(Q^{2}\right)}{2 \pi} P_{q q}(x) \otimes c_{v}\left(x, Q^{2}\right), \\
\frac{d c_{\text {sea }}\left(x, Q^{2}\right)}{d t} & =\frac{\alpha_{s}\left(Q^{2}\right)}{2 \pi}\left[P_{q q}(x) \otimes c_{s e a}\left(x, Q^{2}\right)+P_{c g}\left(x, Q^{2}\right) \otimes g\left(x, Q^{2}\right)\right],
\end{aligned}
$$

The massive-quark splitting functions $P_{c g}\left(x, Q^{2}\right)$ and $P_{c \gamma}\left(x, Q^{2}\right)$ are singular at the charm threshold $x=1 / a$. Due to this singularity, we find that much CPU time is needed in order to get an accurate numerical solution when one solves Eqs.(4.3a), (4.3b), (4.6a) and (4.6b) directly. The numerical problem associated with the use of the massive-quark splitting function of [27] is severer in the photon structure than in the proton structure because of the presence of the leading inhomogeneous term. We can avoid, however, appearance of the singular massive splitting functions, and obtain a set of equations which contain only smooth functions by dividing further the valence- and sea- charm-quark distributions into the QPM part and the remnant. The QPM part of the valence-charm-quark distribution is defined by Eq.4.2a) and that of the sea-charm-quark distribution is defined by Eq.(4.2b). The remnants are defined through the following equations:

$$
\begin{gathered}
c_{v}\left(x, Q^{2}\right) \equiv c_{v}^{\mathrm{QPM}}\left(x, Q^{2}\right)+\delta c_{v}\left(x, Q^{2}\right), \\
c_{\text {sea }}\left(x, Q^{2}\right) \equiv c_{\text {sea }}^{\mathrm{QPM}}\left(x, Q^{2}\right)+\delta c_{\text {sea }}\left(x, Q^{2}\right) .
\end{gathered}
$$

Using Eqs.(4.2), (4.4), and (4.6), one can derive the following equations that govern the deviations $\delta c_{v}$ and $\delta c_{\text {sea }}$ : 


$$
\begin{aligned}
\frac{d}{d t} \delta c_{v}\left(x, Q^{2}\right) & =\frac{\alpha_{s}\left(Q^{2}\right)}{2 \pi} P_{q q}(x) \otimes\left[c_{v}^{\mathrm{QPM}}\left(x, Q^{2}\right)+\delta c_{v}\left(x, Q^{2}\right)\right], \\
\frac{d}{d t} \delta c_{\text {sea }}\left(x, Q^{2}\right) & =\frac{\alpha_{s}\left(Q^{2}\right)}{2 \pi} P_{q q}(x) \otimes\left[c_{\text {sea }}^{\mathrm{QPM}}\left(x, Q^{2}\right)+\delta c_{\text {sea }}\left(x, Q^{2}\right)\right],
\end{aligned}
$$

within the similar approximation that Glück, Hoffmann and Reya made [27]. Here we take the boundary conditions

$$
\delta c_{v, \text { sea }}\left(x, Q_{0}^{2}\right)=0
$$

Note that there now appear no massive splitting functions in these equations. Intuitively, this is because, the deviation of the effective charm quark distribution from the QPM prediction is caused by the gluon emission from the charm quarks, and the emission is approximated by the massless quark splitting function $P_{q q}(x)$ in the scheme of Ref. [27]. A brief explanation of the numerical method which we employ to solve these equations is given in Appendix A.

The resulting effective charm-quark distributions (multiplied by $x$ ) in the photon are shown in Fig. 5 for $Q^{2}=4,20$ and $100 \mathrm{GeV}^{2}$, where the boundary conditions (4.9) are set at $Q_{0}^{2}=4 \mathrm{GeV}^{2}$. Also shown in the figure for comparison are the valence-up-quark distributions of WHIT1-3 and WHIT4-6. Predictions of the QPM are shown by solid lines while those of the massive-quark inhomogeneous AP equations are shown by dash-dotted lines. We find that the differences between the predictions of the two approaches are negligibly small for the sea-charm-quark distribution in WHIT1 to WHIT6. From Fig. 5, we find that the QPM prediction for the valence-charm-quark distribution differs by up to about $20 \%$ at $Q^{2}=100 \mathrm{GeV}^{2}$. The shape of the prediction in the massive inhomogeneous $\mathrm{AP}$ equations is softer than the QPM one as expected. Since the massless splitting function $P_{q q}$ is used for describing the gluon emission from the charm quark, the deviation from the QPM predictions in the lower $Q^{2}$ region may be an overestimate.

From the above discussion, we conclude that the QPM calculation of the effective charm quark distribution is appropriate in the region $Q^{2} \lesssim 100 \mathrm{GeV}^{2}$, given the present experimental accuracy and theoretical uncertainties in the charm quark mass and higher order corrections. At sufficiently high $Q^{2}$, the massless 4-flavor AP equations should become a 
good approximation. However, we find that the charm quark threshold effect is still significant near $x \sim 1$ even at $Q^{2} \sim 100 \mathrm{GeV}^{2}$. We therefore use the massive inhomogeneous $\mathrm{AP}$ equations between $Q^{2}=100 \mathrm{GeV}^{2}$ and $Q^{2}=2500 \mathrm{GeV}^{2}$ up to where we give parametrizations of the parton distributions. We require the continuity of all effective parton distributions at $Q^{2}=100 \mathrm{GeV}^{2}$ : at $4 \mathrm{GeV}^{2} \leq Q^{2} \leq 100 \mathrm{GeV}^{2}$ we use the massless $n_{f}=3$ inhomogeneous AP equations with the QPM approximation to the charm quark distribution, and at 100 $\mathrm{GeV}^{2} \leq Q^{2} \leq 2500 \mathrm{GeV}^{2}$ we use the massive inhomogeneous AP equations with the massive charm quark. The boundary conditions (4.9) are hence set at $Q_{0}^{2}=100 \mathrm{GeV}^{2}$.

Finally, as is mentioned in Sec. [IC, the effective bottom quark distribution in the photon may be approximated by the QPM calculations $\left(\gamma^{*} \gamma \rightarrow b \bar{b}\right.$ and $\left.\gamma^{*} g \rightarrow b \bar{b}\right)$ all the way up to $Q^{2} \simeq 2500 \mathrm{GeV}^{2}$, and hence we do not give parametrizations of the effective b-quark distribution in the photon.

\section{CHARM PRODUCTION CROSS SECTION}

In this section we study the charm-quark production cross section via the two-photon processes by using our new effective parton distribution functions in the photon. The charm quark production cross section is expected to be much more sensitive on the gluonic content of the photon than the photon structure function [23].

\section{A. Equivalent real photon approximation}

To calculate the charm-quark production cross section in the two-photon processes, we employ the equivalent real photon approximation (EPA) to the nearly on-shell virtual photons [5, 37].

In the EPA the charm-quark pair production cross section for the process $e^{+} e^{-} \rightarrow$ $e^{+} e^{-} c \bar{c} X$ is approximated by the convolution of the effective real photon fluxes in the $e^{ \pm}$ beam and the cross section of the subprocess $\gamma \gamma \rightarrow c \bar{c} X$ : 


$$
\sigma\left(e^{+} e^{-} \rightarrow e^{+} e^{-} c \bar{c} X\right) \cong \int d x_{1} d x_{2} D_{\gamma / e}\left(x_{1}, Q_{1}^{2}\right) D_{\gamma / e}\left(x_{2}, Q_{2}^{2}\right) \hat{\sigma}\left(\gamma_{1} \gamma_{2} \rightarrow c \bar{c} X\right)
$$

where $\hat{\sigma}\left(\gamma_{1} \gamma_{2} \rightarrow c \bar{c} X\right)$ is the subprocess cross section at $W^{2}=s x_{1} x_{2}$, and $D_{\gamma / e}\left(x_{i}, Q_{i}^{2}\right) \quad(i=$ $1,2)$ is the equivalent real photon distribution in the electron $(i=1)$ or in the positron $(i=2)$. The c.m. energy of the colliding $e^{-} e^{+}$is $\sqrt{s}$, and that of the colliding two photons is $W$. The improved form of the photon distribution is written as [38]

$$
D_{\gamma / e}\left(x, Q^{2}\right)=\frac{\alpha}{2 \pi} \frac{1+(1-x)^{2}}{x}\left[\log \frac{Q^{2}}{t_{\min }}-1\right]+\frac{\alpha}{2 \pi} x,
$$

where $t_{\text {min }}=m_{e}^{2} x^{2} /(1-x)$ is the kinematical limit of the magnitude of the lepton momentum transfer $t$ and the second term in the right-hand-side denotes the contribution of the electron helicity flip amplitudes 38]. The scale $Q^{2}$ should be set by the dynamical condition that the subprocess "cross section" for a virtual process is damped at $|t|>Q^{2}$ [37.

We choose the scales $Q_{i}^{2}$ as follows. when the photon $\left(\gamma_{i}\right)$ couples directly to the cham quark, we set

$$
Q_{i}^{2}=\min \left[m_{c}^{2}+p_{t}^{2}, t_{\max }, t_{\text {cut }}\right]
$$

where $p_{t}$ is the transverse momentum of the charm quark in the $\gamma \gamma$ c.m. system and $t_{\max }=$ $s\left(1-x_{i}\right)$ is the kinematical maximum of the momentum transfer, and $t_{\text {cut }}$ denotes the possible experimental cut on the magnitude of the momentum transfer. The scale $m_{c}^{2}+p_{t}^{2}$ can be interpreted as the virtuality of the internal charm quark line; if the virtuality of the photon is larger than that of the internal charm quark, the production of charm quark is strongly suppressed. If the photon $\gamma_{i}$ resolves into light partons that contribute to the $c \bar{c}$ production subprocesses, we set

$$
Q_{i}^{2}=\min \left[2 \mathrm{GeV}^{2}, t_{\max }, t_{\text {cut }}\right]
$$

since the gluon content of the photon should be suppressed if its virtuality is much larger than the hadronic scale. Although in principle the quark and gluon contents of a sufficiently virtual photon is calculable in perturbative QCD, we neglect contributions from virtual photons with $\left|t_{i}\right|>2 \mathrm{GeV}^{2}$. The uncertainty associated with the choice of the cut-off scale 
may be estimated by changing the scale by a factor of two, and it is at a few $\%$ level at TRISTAN/LEP energies, while it can be reduced significantly by reducing the $t_{\text {cut }}$ value by an anti-tagging.

To check the validity of our approximation Eq. (5.1) with the scale choice Eq. (5.3), we compare the EPA prediction with the exact cross section for the direct process $e^{+} e^{-} \rightarrow$ $e^{+} e^{-} c \bar{c}$ in which both photons couple directly to the charm quarks. In Fig. 6 we compare differential cross section of charm quark pair production evaluated exactly (solid lines with error bars) with that of the EPA prediction (dotted lines) against the cosine of the scattering angle $\hat{\theta}$ of the subprocess $\gamma^{*} \gamma^{*} \rightarrow c \bar{c}$ in the $c \bar{c}$ rest frame. The exact cross section has been calculated by HELAS [39] with the help of a Monte Carlo integration package BASES 40].

Near the charm quark pair threshold (Fig. 6a) we find almost exact agreements between the EPA and the exact results. At far above the threshold energy (Fig. [b]), the subprocess cross section has peaks at $\cos \hat{\theta}= \pm 1$ where our EPA prediction underestimates the cross section by $\sim 30 \%$. Fig. 7 shows the charm quark pair invariant mass $(\sqrt{\hat{s}})$ distribution after integrating over the scattering angle $\hat{\theta}$. Though there is a tendency that our EPA gives slightly smaller cross section, we find that the EPA calculation of the total cross section agrees with the exact cross section at $1 \%$ level.

\section{B. Charmed particle production cross section}

The inclusive $\gamma \gamma \rightarrow c \bar{c} X$ cross section in Eq. (5.1), including the resolved processes, is described in the leading order as

$$
\begin{aligned}
d \hat{\sigma}(\gamma \gamma \rightarrow c \bar{c} X) & =\left.d \hat{\sigma}(\gamma \gamma \rightarrow c \bar{c})\right|_{\hat{s}=W^{2}} \\
& +\left.2 \int d x g\left(x, Q^{2}\right) d \hat{\sigma}(\gamma g \rightarrow c \bar{c})\right|_{\hat{s}=W^{2} x} \\
& +\left.\int d x d y g\left(x, Q^{2}\right) g\left(y, Q^{2}\right) d \hat{\sigma}(g g \rightarrow c \bar{c})\right|_{\hat{s}=W^{2} x y} \\
& +\left.2 \int d x d y \sum_{i=u, d, s} q_{i}\left(x, Q^{2}\right) \bar{q}_{i}\left(y, Q^{2}\right) d \hat{\sigma}(q \bar{q} \rightarrow c \bar{c})\right|_{\hat{s}=W^{2} x y}
\end{aligned}
$$


where $W$ is the invariant mass of the two photons and $\sqrt{\hat{s}}$ is that of the charm quark pair. The effective gluon and quark distribution functions in the photon are denoted by $g\left(x, Q^{2}\right), q_{i}\left(x, Q^{2}\right)$, for which we use the parametrizations of WHIT1 to WHIT6 parton distributions. The scale $Q^{2}$ of the parton distribution functions and the QCD coupling $\alpha_{s}\left(Q^{2}\right)$ has been chosen as follows: $Q^{2}=m_{c}^{2}+p_{t}^{2}$ for $\gamma g \rightarrow c \bar{c}$ and $g g \rightarrow c \bar{c}$, and $Q^{2}=\hat{s}$ for $q \bar{q} \rightarrow c \bar{c}$.

We show in Fig. \& the total charmed particle production cross sections. The six curves are obtained by setting $m_{c}=1.5 \mathrm{GeV}, \alpha=1 / 137$ and $\Lambda_{4}=0.4 \mathrm{GeV}$, and by imposing the open charm production cut $W \geq 2 m_{D}=3.74 \mathrm{GeV}$. The vertical bars attached to the WHIT1, WHIT4 and WHIT6 curves indicate typical uncertainties in the cross section on the charm quark mass $m_{c}$ between $1.3 \mathrm{GeV}$ and $1.7 \mathrm{GeV}$. It is notable that uncertainty due to the charm quark mass is not reduced at high energies, because the total cross section is always dominated by the charm-quark pair production near the threshold. Higher order QCD corrections [41,31] also modify the cross sections.

As can be seen from Fig. \&, the total charm quark production cross sections at high energy $e^{+} e^{-}$collider experiments are sensitive to the gluon distribution in the photon, which cannot be measured accurately at the current photon structure function experiments as we describe in Sec. [II. At TRISTAN energies, the predictions using WHIT1 to WHIT3 and those using WHIT4 to WHIT6 are almost the same within each group. At LEP and LEP200 energies, the total charmed particle pair production cross section of the two photon process is more sensitive to the small $x$ behavior of the gluon distribution, and WHIT1 to WHIT6 predictions can be distinguished provided that the uncertainties due to the charm quark mass and higher order corrections are reduced.

Fig. 9 shows the total cross sections for the process $e^{+} e^{-} \rightarrow e^{+} e^{-} c \bar{c} X$, together with the individual contribution of the direct photon process (the first term of Eq. (5.5)), the once-resolved photon processes (the second term of Eq. (5.5)), and the twice-resolved photon processes (the third and the last terms of Eq. (5.5)). From Fig. 9, we find that the resolved 
processes, which are governed mainly by the gluon contents of the photon, grows much more rapidly for WHIT6 than for WHIT1. The contribution from the once-resolved processes overcomes that of the direct process even at TRISTAN energies for WHIT6, while they become comparable only at around LEP200 energy for WHIT1. Hence the energy dependence of the once-resolved photon contribution to the charm quark pair production cross section will be useful to determine the gluon distribution.

\section{CONCLUSIONS}

We have studied all the available photon structure function data [11 [17,24, 26] at 4 $\mathrm{GeV}^{2}<Q^{2}<100 \mathrm{GeV}^{2}$ in the leading order of perturbative $\mathrm{QCD}$, and have found a new set of the effective scale-dependent parton distributions in the photon, WHIT1 to WHIT6, which are all consistent with the present data (Fig. 1). The six parton distributions have systematically different gluon contents (Fig. 4) and their parametrizations are given in Table IV to [X]. We have studied carefully the charm quark contributions to the observed structure functions, which are evaluated by using the lowest order quark parton model matrix elements $\left(\gamma^{*} \gamma \rightarrow c \bar{c}\right.$ and $\left.\gamma^{*} g \rightarrow c \bar{c}\right)$, and by using the massive inhomogeneous AP equations. We have found that the photon structure function has poor sensitivity to the gluon distribution, except at very small $x$. In order to probe the gluon content of the photon from the photon structure function at small $x$, a careful analysis of experimental data is needed.

Predictions have also been given for the total charm quark pair production cross section in the two-photon collision process including the resolved processes at $e^{+} e^{-}$colliders. At PEP and PETRA energies, the difference in the predictions of WHIT1 to WHIT6 distributions is badly observable, while at TRISTAN energies WHIT4 to WHIT6 distributions predict significantly higher cross section than WHIT1 to WHIT3 (Fig. 8). For all of our parton distributions, WHIT1 to WHIT6, the contribution of the once-resolved process exceeds that of the direct process at energies above around $200 \mathrm{GeV}$ (Fig. 9). 


\section{ACKNOWLEDGMENT}

We are grateful to our experimental colleagues, T. Nozaki of AMY, T. Tauchi and H. Hayashii of TOPAZ and S. Odaka, H. Ohyama, T. Oyama and S. Uehara of VENUS collaborations, for keeping us informed about the latest analysis of their data. We thank M. Drees and P. M. Zerwas for valuable discussions on the charm quark production cross

section. We also thank J. Kanzaki for discussions and S. Matsumoto for providing us with an efficient computer program to perform the $\chi^{2}$ fit. T.I. and I.W. wish to thank Japan Society for the Promotion of Science for the fellowship. The work of I.W. is partially supported by the Grant-in-Aid for Scientific Research from the Ministry of Education, Science and Culture of Japan.

\section{APPENDIX A: THREE METHODS TO SOLVE THE AP EQUATIONS}

In this appendix we introduce three numerical methods for solving the massless inhomogeneous AP equations of Eq. (2.1) and the massive inhomogeneous AP equations of Eq. (4.3). We first introduce the Mellin transformation technique in Sec. A1, and in Sec. A2 we describe the recursion method that we actually use in performing the $\chi^{2}$ fit to the experimental data. We use the Runge-Kutta method to solve the massive inhomogeneous AP equations, which is discussed in Sec. A3.

The first two methods, the Mellin transformation and the recursion method, work only for the massless AP equations, while the Runge-Kutta method can be applicable to both the massive AP equations and the massless ones. We use all these three methods for cross checking of our numerical results for the massless AP equations.

\section{Moment method for the massless inhomogeneous AP equations}

The Mellin transformation $\tilde{f}(n)$ of $f(x)$ is defined as

$$
\tilde{f}(n) \equiv \int_{0}^{1} d x x^{n-1} f(x)
$$


where $n$ is a complex number. This transformation solves the convolution integrals in the inhomogeneous AP equations as simple products of the Mellin transforms of the splitting function and the parton distribution function:

$$
\int_{0}^{1} d x x^{n-1} P(x) \otimes q(x)=\tilde{P}(n) \tilde{q}(n) .
$$

The inhomogeneous AP equations with $n_{f}$ massless quarks

$$
\begin{aligned}
& \frac{d q_{i}\left(x, Q^{2}\right)}{d t}=\frac{\alpha}{2 \pi} e_{i}^{2} P_{q \gamma}(x)+\frac{\alpha_{s}\left(Q^{2}\right)}{2 \pi}\left[P_{q q}(x) \otimes q_{i}\left(x, Q^{2}\right)+P_{q g}(x) \otimes g\left(x, Q^{2}\right)\right], \\
& \frac{d g\left(x, Q^{2}\right)}{d t}=\frac{\alpha_{s}\left(Q^{2}\right)}{2 \pi}\left[P_{g q}(x) \otimes 2 \sum_{i=1}^{n_{f}} q_{i}\left(x, Q^{2}\right)+P_{g g}\left(x, n_{f}\right) \otimes g\left(x, Q^{2}\right)\right],
\end{aligned}
$$

with the splitting functions

$$
\begin{aligned}
& \left.P_{q q}(z)=\frac{4}{3}\left[\frac{1+z^{2}}{(1-z)_{+}}+\frac{3}{2} \delta(1-z)\right]\right], \\
& P_{g q}(z)=\frac{4}{3} \frac{1+(1-z)^{2}}{z}, \\
& P_{q g}(z)=\frac{1}{2}\left[z^{2}+(1-z)^{2}\right], \\
& P_{g g}(z)=6\left[\frac{z}{(1-z)_{+}}+\frac{(1-z)}{z}+z(1-z)+\left(\frac{11}{12}-\frac{n_{f}}{18}\right) \delta(1-z)\right], \\
& P_{q \gamma}(z)=6 P_{q g}(z),
\end{aligned}
$$

are transformed to the following form in the $n$-space:

$$
\begin{aligned}
& \frac{d \tilde{q}_{i}\left(n, Q^{2}\right)}{d t}=\frac{\alpha}{2 \pi} e_{i}^{2} \tilde{P}_{q \gamma}(n)+\frac{\alpha_{s}\left(Q^{2}\right)}{2 \pi}\left[\tilde{P}_{q q}(n) \tilde{q}_{i}\left(n, Q^{2}\right)+\tilde{P}_{q g}(n) \tilde{g}\left(n, Q^{2}\right)\right], \\
& \frac{d \tilde{g}\left(n, Q^{2}\right)}{d t}=\frac{\alpha_{s}\left(Q^{2}\right)}{2 \pi}\left[2 \sum_{j=1}^{n_{f}} \tilde{P}_{g q}(n) \tilde{q}_{j}\left(n, Q^{2}\right)+\tilde{P}_{g g}\left(n, n_{f}\right) \tilde{g}\left(n, Q^{2}\right)\right],
\end{aligned}
$$

with

$$
\begin{aligned}
& \tilde{P}_{q q}(n)=\frac{8}{3}\left[\frac{3}{4}-\frac{2 n+1}{2 n(n+1)}-\gamma-\Psi(n)\right], \\
& \tilde{P}_{g q}(n)=\frac{4}{3} \frac{n^{2}+n+2}{n\left(n^{2}-1\right)} \\
& \tilde{P}_{q g}(n)=\frac{n^{2}+n+2}{2 n(n+1)(n+2)}, \\
& \tilde{P}_{g g}(n)=6\left[\frac{11}{12}-\frac{n_{f}}{18}+\frac{2-n}{n(n-1)}+\frac{1}{(n+1)(n+2)}-\gamma-\Psi(n)\right], \\
& \tilde{P}_{q \gamma}(n)=6 \tilde{P}_{q g}(n) .
\end{aligned}
$$


Here $\Psi(x) \equiv d \log \Gamma(x) / d x$ is the di-gamma function and $\gamma=0.577 \cdots$ is Euler constant. Eq. (A5) can be readily solved analytically by diagonalizing with respect to the parton flavors, and we find $\tilde{q}_{i}\left(n, Q^{2}\right)$ and $\tilde{g}\left(n, Q^{2}\right)$ at arbitrary $Q^{2}>Q_{0}^{2}$.

The $x$-space solution, $q_{i}\left(x, Q^{2}\right)$, can then be obtained by performing the inverse Mellin transformation

$$
q_{i}\left(x, Q^{2}\right)=\frac{1}{2 \pi i} \int_{C} d n x^{-n} \tilde{q}_{i}\left(n, Q^{2}\right)
$$

numerically. The complex integration path $C$ must be in the right half plane of all singularities of the integrands. We choose the path 29]

$$
C=C_{+}-C_{-}, \quad C_{ \pm}: n=2.5+\exp ( \pm 3 \pi i / 4) u,(u \text { goes from } 0 \text { to } \infty) .
$$

The angle of this path, $3 \pi / 4$, makes the integrals converge. For each value of $x$ in Eq. (A7), $\left|x^{-n}\right|=|\exp (-n \log (x))| \sim \exp (\log (x) u / \sqrt{2})$ can be neglected at sufficiently large $u$ e.g. $u \gtrsim-25 / \log (x)$, for slowly varying $n$-space functions, $\tilde{q}_{i}\left(n, Q^{2}\right)$.

Although the Mellin transformation method is compact and fast, it is not generally useful, because we have to restrict our input distributions $q_{i}\left(x, Q_{0}^{2}\right), g\left(x, Q_{0}^{2}\right)$ to those functions whose Mellin transformations can be analytically obtained and hence we can not use it to solve for our sea-quark distributions (see Eq.(2.14)), and once the splitting functions $\tilde{P}_{i j}(n)$ have a certain dependence on $Q^{2}$, which is the case in the massive AP equations, we can no longer solve Eq. (A5) analytically in general.

\section{Recursive method for the massless AP equations}

The second method to solve the massless AP equations is based on the power expansion of the solution by the double logarithmic energy scale parameter $s$ :

$$
s \equiv \log \left(t / t_{0}\right)=\log \left[\log \left(Q^{2} / \Lambda^{2}\right) / \log \left(Q_{0}^{2} / \Lambda^{2}\right)\right]
$$

To improve the convergence of the power expansion and the behavior at small $x$, we introduce the rescaled parton distribution $\hat{q}_{i}$, defined as 


$$
\begin{gathered}
\hat{q}_{i}(x, s) \equiv x^{2} e^{-s} q_{i}\left(x, Q^{2}\right), \\
\hat{g}(x, s) \equiv x^{2} e^{-s} g\left(x, Q^{2}\right)
\end{gathered}
$$

The massless inhomogeneous AP equations for $n_{f}$ quark flavors, Eq. (2.1), can then be rewritten as follows:

$$
\begin{aligned}
& \frac{d \hat{q}_{i}(x, s)}{d s}=t_{0} \frac{\alpha}{2 \pi} e_{i}^{2} \hat{P}_{q \gamma}(x)+t_{0} e^{s} \frac{\alpha_{s}\left(Q^{2}\right)}{2 \pi}\left[\hat{P}_{q q}(x) \otimes \hat{q}_{i}(x, s)+\hat{P}_{q g}(x) \otimes \hat{g}(x, s)\right]-\hat{q}_{i}(x, s), \\
& \frac{d \hat{g}(x, s)}{d s}=t_{0} e^{s} \frac{\alpha_{s}\left(Q^{2}\right)}{2 \pi}\left[2 \sum_{i=1}^{n_{f}} \hat{P}_{g q}(x) \otimes \hat{q}_{i}(x, s)+\hat{P}_{g g}\left(x, n_{f}\right) \otimes \hat{g}(x, s)\right]-\hat{g}(x, s),
\end{aligned}
$$

where we define the rescaled splitting functions,

$$
\hat{P}_{i j}(x)=x^{2} P_{i j}(x),
$$

which is regular at $x \rightarrow 0$. Since the QCD running coupling $\alpha_{s}\left(Q^{2}\right)$ scales as $\exp (-s) \sim$ $1 / \log Q^{2}$ in the leading $\log$ approximation, the factor $e^{s} \alpha_{s}\left(Q^{2}\right)$ is regarded to be a constant:

$$
\epsilon=t_{0} e^{s} \frac{\alpha_{s}\left(Q^{2}\right)}{2 \pi}=\frac{6}{33-2 n_{F}}
$$

where $n_{F}$ is the number of light-quark flavors which governs the running of the QCD coupling $\alpha_{s}$. We adopt $n_{F}=4$ for $4.0 \mathrm{GeV}^{2} \leq Q^{2} \leq 100 \mathrm{GeV}^{2}$, and $n_{F}=5$ for $Q^{2}>100 \mathrm{GeV}^{2}$, according to Eq. (2.16).

Integrating Eq. (A11), one finds

$$
\hat{q}_{i}(x, s)=\hat{q}_{i}(x, 0)+s t_{0} \frac{\alpha}{2 \pi} e_{i}^{2} \hat{P}_{i \gamma}(x)+\epsilon \sum_{j} \hat{P}_{i j} \otimes \int_{0}^{s} d s^{\prime} \hat{q}_{j}\left(x, s^{\prime}\right)-\int_{0}^{s} d s^{\prime} \hat{q}_{i}\left(x, s^{\prime}\right),
$$

where $\hat{q}_{i}(x, 0)$ is nothing but the rescaled input parton distribution at $Q^{2}=Q_{0}^{2}$. Expanding the parton distributions by powers of $s$, i.e.,

$$
\hat{q}_{i}(x, s)=\sum_{\ell=0}^{\infty} \frac{s^{\ell}}{\ell !} \hat{q}_{i}^{(\ell)}(x),
$$

one can reduce Eq. (A14) order by order of $s$ :

$$
\begin{aligned}
& \hat{q}_{i}^{(0)}(x)=\hat{q}_{i}(x, 0) \\
& \hat{q}_{i}^{(1)}(x)=t_{0} \frac{\alpha}{2 \pi} e_{i}^{2} \hat{P}_{i \gamma}(x)+\epsilon \sum_{j} \hat{P}_{i j}(x) \otimes \hat{q}_{j}^{(0)}(x)-\hat{q}_{i}^{(0)}(x) \\
& \hat{q}_{i}^{(\ell)}(x)=\epsilon \sum_{j} \hat{P}_{i j}(x) \otimes \hat{q}_{j}^{(\ell-1)}(x)-\hat{q}_{i}^{(\ell-1)}(x) \quad(\text { for } \ell \geq 2) .
\end{aligned}
$$


The above equations give the input parton distribution $\hat{q}_{i}(x, 0)$ as the zero-th order approximation for $\hat{q}_{i}(x, s)$. The first correction which is linear to $s$ is the sum of the inhomogeneous term and the terms which is driven by the zero-th approximation (A16b). The higher order corrections are determined recursively by Eq.(A160). Summing all the contributions as in Eq. (A15) up to an appropriate order, one obtains the solution $\hat{q}_{i}(x, s)$ with a given accuracy.

This method is useful for arbitrary input parton distributions. This is an advantage of this method as compared to the moment method in Sec. A1, since our input sea-quark distribution is calculated by the convolution integral in Eq. (2.14) and hence does not have analytic Mellin transform. Furthermore, we find that the recursive method needs much less CPU time than the more general Runge-Kutta method of Sec. A3. We therefore use this scheme in the actual fitting of the experimental data.

When one solves the homogeneous AP equations, similar method can be used, where the scale parameter $s$ is expressed often by the QCD coupling constant $\alpha_{s}\left(Q^{2}\right)$ as $s \equiv$ $\log \left[\alpha_{s}\left(Q_{0}^{2}\right) / \alpha_{s}\left(Q^{2}\right)\right]$. In this definition, one can naturally incorporate the effect of the change of the effective number of the quark flavors $n_{F}$ at the quark mass thresholds by simply rescaling the $s$ variable. However, due to the presence of the inhomogeneous term, we cannot absorb all scales into the $s$ variable.

Even though the recursive method is powerful for solving the massless inhomogeneous AP equations, it cannot be used to solve the massive AP equations. This is because the significant threshold effect in the massive quark distribution and the singular mass effect in the massive splitting functions do not allow the power expansion like Eq. A15)

\section{Runge-Kutta method}

The Runge-Kutta method is the general method to solve the differential equations and it requires a relatively large CPU time and sufficiently large number of data points for precise calculation. We use this method to integrate the inhomogeneous AP equations with a massive charm quark: 


$$
\begin{aligned}
& \frac{d q_{i}\left(x, Q^{2}\right)}{d t}=\frac{\alpha}{2 \pi} e_{i}^{2} P_{q \gamma}(x)+\frac{\alpha_{s}\left(Q^{2}\right)}{2 \pi}\left[P_{q q}(x) \otimes q_{i}\left(x, Q^{2}\right)+P_{q g}(x) \otimes g\left(x, Q^{2}\right)\right] \\
& \frac{d g\left(x, Q^{2}\right)}{d t}=\frac{\alpha_{s}\left(Q^{2}\right)}{2 \pi}\left[P_{g q}(x) \otimes 2\left(\sum_{i=u, d, s} q_{i}\left(x, Q^{2}\right)+c\left(x, Q^{2}\right)\right)+P_{g g}(x, 3) \otimes g\left(x, Q^{2}\right)\right] \\
& \frac{d c\left(x, Q^{2}\right)}{d t}=\frac{\alpha}{2 \pi} e_{c}^{2} P_{c \gamma}\left(x, Q^{2}\right)+\frac{\alpha_{s}\left(Q^{2}\right)}{2 \pi}\left[P_{q q}(x) \otimes c\left(x, Q^{2}\right)+P_{c g}\left(x, Q^{2}\right) \otimes g\left(x, Q^{2}\right)\right] .
\end{aligned}
$$

After introducing the QPM component of the charm quark distribution by Eq. (4.2), the deviations from the QPM predictions as defined in Eq. (4.7) satisfy the following equations:

$$
\begin{aligned}
\frac{d}{d t} \delta c_{v}\left(x, Q^{2}\right) & =\frac{\alpha_{s}\left(Q^{2}\right)}{2 \pi} P_{q q}(x) \otimes\left[c_{v}^{\mathrm{QPM}}\left(x, Q^{2}\right)+\delta c_{v}\left(x, Q^{2}\right)\right], \\
\frac{d}{d t} \delta c_{\text {sea }}\left(x, Q^{2}\right) & =\frac{\alpha_{s}\left(Q^{2}\right)}{2 \pi} P_{q q}(x) \otimes\left[c_{\text {sea }}^{\mathrm{QPM}}\left(x, Q^{2}\right)+\delta c_{\text {sea }}\left(x, Q^{2}\right)\right] .
\end{aligned}
$$

It is the above two equations with the boundary condition Eq. (4.9) that we solve by using the Runge-Kutta method. It is difficult to apply the previous two methods, the Mellin transformation method or the recursion method, to solve the above equations. For the Mellin transformation method, we can not analytically Mellin transform $c_{v, \text { sea }}^{\mathrm{QPM}}$. For the recursive method of Sec. A2, the expansion of $w(z, r)$ in powers of $s$ fails at the threshold $z=1 / a=1 /\left(1+4 m_{c}^{2} / Q^{2}\right)$.

To solve the massive AP equations of Eqs. (A17a), (A17b), (A18) we use the fourth order Runge-Kutta method with adaptive step size control for calculating the evolution of t. By discretizing the $x$ variable as $x_{j}(j=0,1 \cdots, N)$, we solve the AP equations as a set of $5(N+1)$ ordinary differential equations, while performing the convolution integral $P(x) \otimes q(x)$ by the Simpson integration. We choose

$$
\begin{aligned}
x_{j} & =\tanh (\sinh (-2+0.04 j)) / 2+1 / 2, \quad(j=0, \cdots, 110), \\
x_{111} & =1,
\end{aligned}
$$

so that the data points are dense near the both ends $x \sim 0$ and $x \sim 1$. We find that $N=111$ is sufficient to archive an accuracy of $1 \%$. 


\section{APPENDIX B: WHIT1-WHIT6 PARAMETRIZATIONS}

For practical use of the WHIT parton distributions which are given by the standard valence-quark input parameters of Eq. (3.2) and the various gluon input parameters as listed in Table III, we give their convenient parametrizations (or simple prescriptions to calculate them). The parametrizations are given in three different $Q^{2}$ regions, $4 \mathrm{GeV}^{2} \leq$ $Q^{2} \leq 100 \mathrm{GeV}^{2}, 100 \mathrm{GeV}^{2} \leq Q^{2} \leq 2500 \mathrm{GeV}^{2}$, and $Q^{2} \leq 4 \mathrm{GeV}^{2}$. The FORTRAN code for the distributions that we use for generating curves in this paper is obtainable from kaoru@kekvax.kek.jp or from kaoru@jpnkekvx.bitnet

\section{Parametrizations in the region $4 \mathrm{GeV}^{2} \leq Q^{2} \leq 100 \mathrm{GeV}^{2}$}

In this $Q^{2}$ region, we parametrize those solutions of the massless 3-flavor AP equations which are described in Sec. IIIB. The heavy quark distributions are calculated by the quark parton model as is mentioned in Sec. IV. Therefore, it is sufficient to give parametrizations for the valence-quark, the gluon and the sea-quark distributions.

The valence-quark distribution is parametrized in the following functional form:

$$
x q_{v}(x, s) / \alpha=\left(A_{0}+A_{1} x+A_{2} x^{2}\right) x^{B}(1-x)^{C},
$$

where $s$ is defined in Eq. (A9) with $Q_{0}^{2}=4 \mathrm{GeV}^{2}$ and $\Lambda=\Lambda_{4}=400 \mathrm{MeV}$, and $A_{i}$ 's, $B$ and $C$ are polynomials of $s$ of at most 4 -th order. The coefficients of these polynomials can be

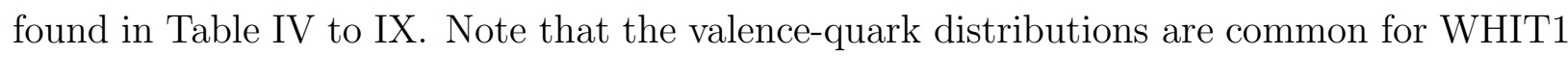
to WHIT3 and for WHIT4 to WHIT6.

For the gluon distribution, we take the following form:

$$
x g(x, s) / \alpha=A x^{B}(1-x)^{C}+\left(A_{0}^{\prime}+A_{1}^{\prime} x\right) x^{B^{\prime}}(1-x)^{C^{\prime}} .
$$

As in the case of the valence-quark, $A$ 's, $B$ 's, and $C^{\prime}$ 's are polynomials of $s$ of at most 4-th

order. The coefficients of these polynomials are also found in Table $\amalg$ to $\amalg$. Note that the second term in Eq. (B2) is common for WHIT1 to WHIT3 and for WHIT4 to WHIT6. The 
reason is that it approximately represents the contribution of the gluons emitted from the valence-quark, and that the valence-quark distribution is common for WHIT1 to WHIT3 and for WHIT4 to WHIT6.

Since the input valence-quark distributions are much harder than the input gluon distributions for the WHIT parton distributions as seen in Eq. (3.2) and Table [1], the contribution of the common part of the gluon distributions is expected to be significant at $x \sim 1$. In fact, we can estimate the behavior of the common part from Eq. (2.60). In the one gluon emission approximation, we get the following relation for the common gluon distribution which originates from the valence-quark:

$$
g(x) \sim P_{g q}(x) \otimes q_{v}(x)
$$

Assuming the behavior of the valence-quark distribution at $x \sim 1$ as $q_{v}(x) \sim(1-x)^{C_{v}}$, Eq.(B3) leads to

$$
g(x) \sim(1-x)^{C_{v}+1} \quad \text { as } \quad x \rightarrow 1
$$

This common part of the gluon distributions is dominant at $x \sim 1$ in the WHIT parton distributions, because $C_{v}<1$ and $C_{g} \geq 3$ for all the input parton distributions. One may find that the above crude estimation of the behavior of the common part of the gluon distributions at $x \sim 1$ works rather well even in quantitative sense by looking at the relevant entries of Table IV to IX.

As for the sea-quark distribution, we parametrize it by the following functional form:

$$
x q_{\text {sea }}(x, s) / \alpha=A x^{\left(B_{0}+B_{1} x\right)}(1-x)^{C} .
$$

The coefficients that describe the $s$ dependence of $A, B$ 's and $C$ are also listed in Table $\amalg$ to $[\mathrm{X}$.

The effective charm quark distribution is calculated by the quark parton model by using Eqs. (4.1) and (4.2). In the calculation of the sea-charm-quark distribution in Eq. (4.2b), the above parametrization of the gluon distribution for each WHIT parton distribution is used. 
An efficient integration routine for the convolution in the calculation of the sea-charm-quark distribution is included in our FORTRAN code for WHIT parametrizations.

Our parametrizations reproduce the distributions within $5 \%$ in the region of $0.001 \leq$ $s \leq 0.8$ (corresponding to the region of $4.01 \mathrm{GeV}^{2} \leq Q^{2} \leq 207 \mathrm{GeV}^{2}$ ) and $0.001 \leq x \leq 0.99$ for the up, down and gluon distributions. Note that the up and down quark distributions are written in terms of the valence- and sea- quark distributions as shown in Eq.(2.5). In the region $0 \leq s \leq 0.001\left(4 \mathrm{GeV}^{2} \leq Q^{2} \leq 4.01 \mathrm{GeV}^{2}\right)$, they do not agree precisely with the distributions mainly because our over-simplified initial distributions do not satisfy the perturbative relations among quarks and gluon distributions such as Eq. (B4). As for the charm quark distributions, our convolution integral routine is sufficiently accurate that their error is at most $5 \%$ reflecting the error in the parametrizations of the gluon distributions.

\section{Parametrizations in the region $100 \mathrm{GeV}^{2} \leq Q^{2} \leq 2500 \mathrm{GeV}^{2}$}

In this $Q^{2}$ region, we parametrize the solutions of the massive inhomogeneous AP equations obtained in Sec. $\mathbb{D}$, in which the charm quark mass is retained. According to the prescription of Sec. IV, we give the deviations from the QPM predictions for the valencecharm-quark and the sea-charm-quark distributions that are defined in Eq. (4.7), in addition to the valence-light-quark, the gluon and the sea-light-quark distributions.

The valence-light-quark, the gluon and the sea-light-quark distributions are parametrized in exactly the same manner as in the previous lower $Q^{2}$ case, except that $Q_{0}$ and $\Lambda$ in the definition of $s$ are now changed to $Q_{0}^{2}=100 \mathrm{GeV}^{2}$ and $\Lambda=\Lambda_{5}=302.3 \mathrm{MeV}$.

The deviation in the valence-charm-quark distribution is parametrized as

$$
x \delta c_{v}(x, s) / \alpha=\left(A_{0}+A_{1} x+A_{2} x^{2}+A_{3} x^{3}\right) x^{B}(1-x)^{C} .
$$

Note that, as seen from Eq. (4.8a), $\delta c_{v}$ is completely universal, i.e. it is common for all six WHIT parton distributions. The parametrization of the deviation in the sea-charm-quark distribution takes the following form: 


$$
x \delta c_{\text {sea }}(x, s) / \alpha=A x^{\left(B_{0}+B_{1} x\right)}(1-x)^{C},
$$

which is the same form as the parametrization of the sea-light-quark distribution. The total charm quark distribution is given by Eqs. (4.5), (4.7). The QPM part of the sea-charmquark distribution is calculated by using the parametrization of the corresponding gluon distribution.

The relative error of these parametrizations is less than $5 \%$ for the up, down and gluon distributions in the region of $0 \leq s \leq 0.4$ (corresponding to the region of $100 \mathrm{GeV}^{2} \leq Q^{2} \leq$ $3120 \mathrm{GeV}^{2}$ ), and $0.00125 \leq x \leq 0.99$. Note that the up and down quark distributions are related to the valence- and the sea- light-quark distributions by Eq. (2.5). For the charm quark distributions, the accuracy is also within $5 \%$ in the same $Q^{2}$ region but in the different $x$ region: $0.00125 \leq x \leq 0.99 / a$, where $x=1 / a=1 /\left(1+4 m_{c}^{2} / Q^{2}\right)$ represents the threshold for the charm quark.

\section{Prescription in the region of $Q^{2} \leq 4 \mathrm{GeV}^{2}$}

Finally, we give a prescription for the parton distributions at $Q^{2} \leq Q_{0}^{2}=4 \mathrm{GeV}^{2}$. This is because occasionally one wants to estimate the effects at lower $Q^{2}$ region. We give a very crude estimate here that can be used in such cases, rather than setting all the distributions to zero or freezing the scale-dependences.

As is explained in the main text, we give initial parton distributions at $Q^{2}=Q_{0}^{2}=4 \mathrm{GeV}^{2}$ and evolve them to higher $Q^{2}$ by using the inhomogeneous AP equations. Since the AP equations cannot generally be solved in the backward direction due to its instability, there are no ways to calculate the correct parton distributions below $4 \mathrm{GeV}^{2}$.

Our prescription to estimate the parton distributions in the region of $Q^{2} \leq 4 \mathrm{GeV}^{2}$ is simply to multiply the factor $\log \left(Q^{2} / \Lambda_{4}^{2}\right) / \log \left(4 \mathrm{GeV}^{2} / \Lambda_{4}^{2}\right)$ to the corresponding parton distributions at $Q^{2}=4 \mathrm{GeV}^{2}$ i.e. the input parton distributions. This crude prescription gives a reasonably good estimate for the valence quark distributions, but the resulting gluon 
distribution may not be realistic. Therefore, one has to be careful when using this estimate at $Q^{2}<4 \mathrm{GeV}^{2}$.

In our application, the charm quark pair production process probes the photon structure down to $m_{c}^{2}$, which can take the lowest value of $(1.3 \mathrm{GeV})^{2}=1.69 \mathrm{GeV}^{2}$ for $m_{c}=1.3 \mathrm{GeV}$. We have checked that the effect of modifying the prescription at $Q^{2}<4 \mathrm{GeV}^{2}$ is negligibly small for the total charm quark production cross section. 


\section{REFERENCES}

* Address after April 1994: Department of Physics, Ochanomizu University, Ohtsuka, Bunkyo-ku, Tokyo 112, Japan.

[1] E. Witten, Nucl. Phys. B120, 189 (1977) .

[2] W. A. Bardeen and A. J. Buras, Phys. Rev. D20, 166 (1979); erratum, D21 2041 (1980); D. W. Duke and J. F. Owens, Phys. Rev. D22 2280 (1980); G. Rossi, Phys. Lett. B130 105 (1983); I. Antoniadis and G. Grunberg, Nucl. Phys. B213 445 (1983).

[3] M. Glück and E. Reya, Phys. Rev. D28 2749 (1983).

[4] M. Drees and K. Grassie, Z. Phys. C28 451 (1985).

[5] C. Weizäcker, Z. Phys. 88612 (1934); E. J. Williams, Phys. Rev. 45729 (1934).

[6] J. H. Field, F. Kapusta and L. Piggioli, Phys. Lett. B181 362 (1986); Z. Phys. C36 $121(1987)$.

[7] L. E. Gordon and J. K. Storrow, Z. Phys. C56 307 (1992).

[8] P. Aurenche, P. Chiappetta, M. Fontannaz, J. P. Guillet and E. Pilon, Z. Phys. C56 $589(1992)$.

[9] M. Glück, E. Reya and A. Vogt, Phys. Rev. D46 1973 (1992).

[10] H. Abramowicz, K. Charchuła and A. Levy, Phys. Lett. B269 458 (1991).

[11] PLUTO collab., Ch. Berger et al., Phys. Lett. B142 111 (1984).

[12] PLUTO collab., Ch. Berger et al., Nucl. Phys. B281 365 (1987).

[13] TASSO collab., M. Althoff et al., Z. Phys. C31 527 (1986).

[14] JADE collab., W. Bartel et al., Z. Phys. C24 231 (1984).

[15] TPC/Two-Gamma collab., H. Aihara et al., Z. Phys. C34 1 (1987). 
[16] TPC/Two-Gamma collab., J. S. Steinman, UCLA preprint, UCLA-HEP-88-004 (1988).

[17] AMY collab., T. Sasaki et al., Phys. Lett. B252 491 (1990).

[18] AMY collab., R. Tanaka et al., Phys. Lett. B277, 215 (1992).

[19] TOPAZ collab., H. Hayashii et al., Phys. Lett. B314, 149 (1993).

[20] TOPAZ collab., R. Enomoto et al., KEK Preprint 93-107 (1993).

[21] VENUS collab., S. Uehara et al., KEK Preprint 93-142 (1993).

[22] AMY collab., T. Aso, talk given at The Second Workshop on TRISTAN Physics at High Luminosities, KEK, Tsukuba, Japan, November 24-26, (1993).

[23] M. Drees and R. M. Godbole, Nucl. Phys. B339 355 (1990).

[24] TOPAZ collab., K. Muramatsu, talk given at The Second Workshop on TRISTAN Physics at High Luminosities, KEK, Tsukuba, Japan, November 24-26, (1993). TOPAZ collab., K. Muramatsu et al., KEK Preprint 94-13 (1994).

[25] VENUS collab., T. Oyama et al., private communication.

[26] OPAL collab., R. Akers et al., Z. Phys.C61 (1994) 199-208.

[27] M. Glück, E. Hoffmann, and E. Reya, Z. Phys. C13 119 (1982).

[28] W. A. Bardeen and A. J. Buras, in [2].

[29] M. Glück, E. Reya and A. Vogt, Phys. Rev. D45 3986 (1991).

[30] M. Fontannaz and E. Pilon, Phys. Rev. D45 382 (1992).

[31] E. Laenen, S. Riemersma, J. Smith and W. L. van Neerven, Stony Brook preprint, ITP-SB-93-1 (1993); ITP-SB-93-46 (1993).

[32] R. J. DeWitt, L. M. Jones, J. D. Sullivan, D. E. Willen, and H. W. Wyld, Jr., Phys. Rev. D19 2046 (1979). 
[33] G. Altarelli and G. Parisi, Nucl. Phys. B126 298 (1977); G. Altarelli, Phys. Rep. 811 (1982).

[34] P. Aurenche, R. Baier, M. Fontannaz, M. N. Kienzle-Focacci and M. Werlen, Phys. Lett. B233 517 (1989).

[35] A. J. Buras and K. J. F. Gaemers, Nucl. Phys. B132 249 (1978); J. F. Owens and E. Reya, Phys. Rev. D17 3003 (1978); R. Baier, J. Engels and B. Petersson, Z. Phys. C2 265 (1979); M. Diemoz, F. Ferroni, E. Longo and G. Martinelli, D. W. Duke and J. F. Owens, Phys. Rev. D30 49 (1984). Z. Phys. C39 21 (1988); A. D. Martin, R. G. Roberts and W. J. Stirling, Phys. Rev. D37 1161 (1988); Phys. Lett. B206 327 (1988); Mod. Phys. Lett. A4 1135 (1989); P. N. Harriman, A. D. Martin, R. G. Roberts and W. J. Stirling, Phys. Rev. D42 798 (1990); Phys. Lett. B243 421 (1990); J. Kwiecinski, A. D. Martin, R. G. Roberts and W. J. Stirling, Phys. Rev. D42 3645 (1990); A. D. Martin, R. G. Roberts and W. J. Stirling, Phys. Rev, D43 3648 (1991).

[36] JADE collab., W. Bartel et al., in ref. [14]; V. Blodbel, in Proc. of the CERN School of Computing, Aiguablanca, Spain, September 1984, CERN 85-09, pp. 88, ed. C. Verkerk (1985); A. Bäcker, in Proc. of the 6th International Workshop on Photon-Photon Collisions, Cranlibakken, September 1984, pp. 205, ed. R. L. Lander (World Scientific, Singapore, 1985).

[37] V. M. Budnev, I. F. Ginzburg, G. V. Meledin and V. G. Serbo, Phys. Rep. 15183 (1975).

[38] K. Hagiwara, H. Iwasaki, A. Miyamoto, H. Murayama and D. Zeppenfeld Nucl. Phys. B365 544 (1991).

[39] H. Murayama, I. Watanabe and K. Hagiwara, HELAS: HELicity Amplitude Subroutines for Feynman Diagram Evaluations, KEK Report 91-11 (January, 1992).

[40] S. Kawabata, Comput. Phys. Commun. 41, 127 (1986). 
[41] M. Drees, M. Krämer, J. Zunft and P. M. Zerwas, Phys. Lett. B306 371 (1993). 


\section{TABLES}

TABLE I. The data of $F_{2}^{\gamma}$ adopted in the fit of the valence-quark parameters.

\begin{tabular}{|c|c|c|c|c|}
\hline collider & collab. & $\left\langle Q^{2}\right\rangle\left(\mathrm{GeV}^{2}\right)$ & $x$ bins & Ref. \\
\hline \multirow[t]{6}{*}{ PETRA } & PLUTO & 4.3 & $0.03-0.17,0.17-0.44,0.44-0.80$ & 11 \\
\hline & & 9.2 & $0.06-0.23,0.23-0.54,0.54-0.90$ & 11 \\
\hline & & 45.0 & $0.25-0.50,0.50-0.75,0.75-0.90$ & 12 \\
\hline & TASSO & 23.0 & $0.20-0.40,0.40-0.60,0.60-0.80,0.80-0.98$ & 13 \\
\hline & JADE & 24.0 & $0.10-0.20,0.20-0.40,0.40-0.60,0.60-0.90$ & 14 \\
\hline & & 100.0 & $0.10-0.30,0.30-0.60,0.60-0.90$ & 14 \\
\hline \multirow[t]{2}{*}{ PEP } & $\mathrm{TPC} / 2 \gamma$ & 5.1 & $0.02-0.20,0.20-0.36,0.36-0.74$ & 15 \\
\hline & & 20.0 & $0.196-0.386,0.386-0.611,0.611-0.963$ & 16 \\
\hline \multirow[t]{6}{*}{ TRISTAN } & AMY & 73.0 & $0.125-0.375,0.375-0.625,0.625-0.875$ & 17 \\
\hline & TOPAZ & 5.1 & $0.076-0.20$ & 24 \\
\hline & & 16.0 & $0.15-0.33,0.33-0.78$ & 24 \\
\hline & & 80.0 & $0.32-0.59,0.59-0.98$ & 24 \\
\hline & VENUS & 40.0 & $0.09-0.27,0.27-0.45,0.45-0.63,0.63-0.81$ & 25 \\
\hline & & 90.0 & $0.19-0.37,0.37-0.55,0.55-0.73,0.73-0.91$ & 25 \\
\hline \multirow[t]{2}{*}{ LEP } & OPAL & 5.9 & $0.091-0.283,0.283-0.649$ & 26 \\
\hline & & 14.7 & $0.137-0.324,0.324-0.522,0.522-0.836$ & 26 \\
\hline
\end{tabular}


TABLE II. The minimal $\chi^{2}$ and the valence-quark parameters as obtained by the best fit. Whenever available, we have taken into account correlation in errors. Degree of the freedom of the fit is $47-3=44$.

\begin{tabular}{|c|c|c|c|c|c|c|c|c|}
\hline \multicolumn{2}{|c|}{ gluon } & \multirow{2}{*}{$\begin{array}{c}\text { best fit } \\
\chi^{2}\end{array}$} & \multicolumn{3}{|c|}{ valence-quark parameters } & \multicolumn{3}{|c|}{ correlations } \\
\hline$A_{g}$ & $C_{g}$ & & $A_{v}$ & $B_{v}$ & $C_{v}$ & $\rho\left(A_{v}, B_{v}\right)$ & $\rho\left(A_{v}, C_{v}\right)$ & $\rho\left(B_{v}, C_{v}\right)$ \\
\hline 0.5 & 3 & 51.6 & $0.930(79)$ & $0.50(17)$ & $0.24(25)$ & -0.52 & -0.75 & 0.88 \\
\hline 0.5 & 6 & 53.2 & $0.933(78)$ & $0.51(17)$ & $0.28(26)$ & -0.52 & -0.75 & 0.88 \\
\hline 0.5 & 9 & 54.0 & $0.938(78)$ & $0.49(16)$ & $0.28(25)$ & -0.52 & -0.75 & 0.88 \\
\hline 0.5 & 15 & 54.3 & $0.948(78)$ & $0.44(16)$ & $0.26(25)$ & -0.52 & -0.75 & 0.88 \\
\hline 1.0 & 3 & 54.0 & $0.873(76)$ & $0.77(21)$ & $0.41(29)$ & -0.52 & -0.73 & 0.88 \\
\hline 1.0 & 6 & 58.2 & $0.882(74)$ & $0.77(20)$ & $0.48(30)$ & -0.53 & -0.74 & 0.89 \\
\hline 1.0 & 9 & 60.2 & $0.892(74)$ & $0.71(19)$ & $0.47(29)$ & -0.53 & -0.74 & 0.88 \\
\hline 1.0 & 15 & 60.4 & $0.911(75)$ & $0.61(17)$ & $0.42(28)$ & -0.53 & -0.75 & 0.88 \\
\hline 1.5 & 3 & 59.8 & $0.821(73)$ & $1.11(26)$ & $0.62(34)$ & -0.53 & -0.72 & 0.89 \\
\hline 1.5 & 6 & 67.8 & $0.837(71)$ & $1.05(24)$ & $0.70(34)$ & -0.54 & -0.73 & 0.89 \\
\hline 1.5 & 9 & 70.9 & $0.853(71)$ & $0.95(22)$ & $0.67(33)$ & -0.54 & -0.73 & 0.89 \\
\hline 1.5 & 15 & 70.1 & $0.879(72)$ & $0.79(19)$ & $0.58(31)$ & -0.53 & -0.74 & 0.89 \\
\hline
\end{tabular}

TABLE III. The $\chi^{2}$ values with the standard valence-quark parameters.

\begin{tabular}{|c|c|c|c|c|c|c|c|}
\hline \multirow{2}{*}{ name } & \multicolumn{2}{|c|}{ gluon } & \multirow[b]{2}{*}{$\chi^{2}$} & \multirow[t]{2}{*}{ name } & \multicolumn{2}{|c|}{ gluon } & \multirow[b]{2}{*}{$\chi^{2}$} \\
\hline & $A_{g}$ & $C_{g}$ & & & $A_{g}$ & $C_{g}$ & \\
\hline \multirow{4}{*}{ WHIT1 } & 0.5 & 1 & 50.7 & \multirow{4}{*}{ WHIT4 } & 1.0 & 1 & 54.2 \\
\hline & 0.5 & 2 & 51.1 & & 1.0 & 2 & 55.0 \\
\hline & 0.5 & 3 & 51.6 & & 1.0 & 3 & 56.0 \\
\hline & 0.5 & 4 & 52.2 & & 1.0 & 4 & 57.0 \\
\hline \multirow{9}{*}{ WHIT2 } & 0.5 & 5 & 52.7 & \multirow{9}{*}{ WHIT5 } & 1.0 & 5 & 57.9 \\
\hline & 0.5 & 6 & 53.2 & & 1.0 & 6 & 58.6 \\
\hline & 0.5 & 7 & 53.6 & & 1.0 & 7 & 59.3 \\
\hline & 0.5 & 8 & 54.0 & & 1.0 & 8 & 59.8 \\
\hline & 0.5 & 9 & 54.3 & & 1.0 & 9 & 60.2 \\
\hline & 0.5 & 10 & 54.5 & & 1.0 & 10 & 60.6 \\
\hline & 0.5 & 11 & 54.7 & & 1.0 & 11 & 60.9 \\
\hline & 0.5 & 12 & 54.9 & & 1.0 & 12 & 61.1 \\
\hline & 0.5 & 13 & 55.1 & & 1.0 & 13 & 61.2 \\
\hline \multirow{3}{*}{ WHIT3 } & 0.5 & 14 & 55.2 & \multirow{3}{*}{ WHIT6 } & 1.0 & 14 & 61.3 \\
\hline & 0.5 & 15 & 55.3 & & 1.0 & 15 & 61.4 \\
\hline & 0.5 & 16 & 55.3 & & 1.0 & 16 & 61.5 \\
\hline
\end{tabular}


TABLE IV. Coefficients of the parametrization for WHIT1 parton distribution in the photon.

\begin{tabular}{|c|c|c|c|c|c|c|}
\hline \multicolumn{2}{|c|}{$Q^{2}$} & \multicolumn{5}{|c|}{$4 \mathrm{GeV}^{2} \leq Q^{2} \leq 100 \mathrm{GeV}^{2}$} \\
\hline & & $s^{0}$ & $s^{1}$ & $s^{2}$ & $s^{3}$ & $s^{4}$ \\
\hline \multirow[t]{5}{*}{$q_{v}$} & $A_{0}$ & 1.882 & 1.213 & 0.697 & 0 & 0 \\
\hline & $A_{1}$ & 0 & -2.361 & -1.136 & 0 & 0 \\
\hline & $A_{2}$ & 0 & 0.528 & 2.406 & 0 & 0 \\
\hline & $B$ & 0.500 & 0.02107 & 0.00413 & 0 & 0 \\
\hline & $C$ & 0.2500 & -0.2376 & 0.2018 & -0.0504 & 0 \\
\hline \multirow[t]{7}{*}{$g$} & $A$ & 2.000 & -3.28 & 2.894 & -1.561 & 0.818 \\
\hline & $B$ & 0 & -0.761 & -0.0490 & 0.446 & 0 \\
\hline & $C$ & 3.000 & 1.586 & -0.949 & 2.425 & 0 \\
\hline & $A_{0}^{\prime}$ & 0 & 0.461 & 0.1041 & -0.01753 & -0.2717 \\
\hline & $A_{1}^{\prime}$ & 0 & 0.00968 & -0.417 & -0.395 & 0.843 \\
\hline & $B^{\prime}$ & -0.414 & -0.0606 & 0.2847 & -0.507 & 0 \\
\hline & $C^{\prime}$ & 1.244 & 0.588 & -1.228 & 0.809 & 0 \\
\hline \multirow[t]{4}{*}{$q_{s e a}$} & $A$ & 0.651 & 1.291 & -4.47 & 5.14 & -2.091 \\
\hline & $B_{0}$ & -0.0382 & 0.0901 & -1.356 & 1.582 & -0.644 \\
\hline & $B_{1}$ & 2.084 & 7.74 & -29.70 & 38.6 & -17.05 \\
\hline & $C$ & 7.00 & -16.08 & 46.7 & -57.1 & 23.86 \\
\hline \multicolumn{2}{|c|}{$Q^{2}$} & \multicolumn{5}{|c|}{$100 \mathrm{GeV}^{2} \leq Q^{2} \leq 2500 \mathrm{GeV}^{2}$} \\
\hline & & $s^{0}$ & $s^{1}$ & $s^{2}$ & $s^{3}$ & $s^{4}$ \\
\hline \multirow[t]{5}{*}{$q_{v}$} & $A_{0}$ & 3.058 & 2.474 & 1.002 & 0 & 0 \\
\hline & $A_{1}$ & -2.182 & -4.48 & -0.2251 & 0 & 0 \\
\hline & $A_{2}$ & 1.522 & 4.31 & 1.314 & 0 & 0 \\
\hline & $B$ & 0.517 & 0.0404 & -0.02100 & 0 & 0 \\
\hline & $C$ & 0.1655 & -0.02062 & 0.0536 & 0 & 0 \\
\hline \multirow[t]{7}{*}{$g$} & $A$ & 0.784 & -2.238 & 16.17 & -62.5 & 83.9 \\
\hline & $B$ & -0.403 & -1.307 & 8.78 & -35.8 & 53.5 \\
\hline & $C$ & 4.45 & 1.027 & 44.6 & -160.0 & 181.6 \\
\hline & $A_{0}^{\prime}$ & 0.3010 & 1.275 & -1.563 & 4.10 & -13.37 \\
\hline & $A_{1}^{\prime}$ & -0.1305 & -1.245 & 2.438 & -2.539 & 12.73 \\
\hline & $B^{\prime}$ & -0.489 & 0.955 & -4.40 & 10.22 & -17.13 \\
\hline & $C^{\prime}$ & 1.331 & -0.2481 & 1.950 & -2.072 & 0 \\
\hline \multirow[t]{4}{*}{$q_{\text {sea }}$} & $A$ & 0.625 & -0.589 & 4.18 & -12.06 & 12.57 \\
\hline & $B_{0}$ & -0.2492 & -0.411 & 0.966 & -2.584 & 2.670 \\
\hline & $B_{1}$ & 2.100 & -5.75 & 47.8 & -140.7 & 147.6 \\
\hline & $C$ & 4.78 & 4.86 & -48.9 & 147.7 & -160.2 \\
\hline \multirow[t]{6}{*}{$\delta c_{v}$} & $A_{0}$ & 0 & 0.1219 & 6.20 & -25.04 & 30.98 \\
\hline & $A_{1}$ & 0 & 1.913 & -76.9 & 318. & -392. \\
\hline & $A_{2}$ & 0 & -7.16 & 250.3 & -1062 & 1308. \\
\hline & $A_{3}$ & 0 & 3.19 & -230.1 & 1012. & -1250. \\
\hline & $B$ & 0.499 & 3.47 & -15.26 & 19.67 & 0 \\
\hline & $C$ & 0.329 & 8.24 & -38.0 & 46.3 & 0 \\
\hline \multirow[t]{4}{*}{$\delta c_{\text {sea }}$} & $A$ & 0 & -0.01815 & 0.002043 & -0.00413 & 0 \\
\hline & $B_{0}$ & -0.3086 & -0.2565 & 0.0984 & 0 & 0 \\
\hline & $B_{1}$ & 1.376 & -0.463 & 1.232 & 0 & 0 \\
\hline & $C$ & 3.65 & 0.729 & -7.57 & 7.79 & 0 \\
\hline
\end{tabular}


TABLE V. Coefficients of the parametrization for WHIT2 parton distribution in the photon.

\begin{tabular}{|c|c|c|c|c|c|c|}
\hline \multicolumn{2}{|c|}{$Q^{2}$} & \multicolumn{5}{|c|}{$4 \mathrm{GeV}^{2} \leq Q^{2} \leq 100 \mathrm{GeV}^{2}$} \\
\hline & & $s^{0}$ & $s^{1}$ & $s^{2}$ & $s^{3}$ & $s^{4}$ \\
\hline \multirow[t]{5}{*}{$q_{v}$} & $A_{0}$ & 1.882 & 1.213 & 0.697 & 0 & 0 \\
\hline & $A_{1}$ & 0 & -2.361 & -1.136 & 0 & 0 \\
\hline & $A_{2}$ & 0 & 0.528 & 2.406 & 0 & 0 \\
\hline & $B$ & 0.500 & 0.02107 & 0.00413 & 0 & 0 \\
\hline & $C$ & 0.2500 & -0.2376 & 0.2018 & -0.0504 & 0 \\
\hline \multirow[t]{7}{*}{$g$} & $A$ & 5.00 & -14.99 & 26.17 & -25.30 & 10.12 \\
\hline & $B$ & 0 & -0.937 & 0.410 & 0.0339 & 0 \\
\hline & $C$ & 9.00 & 0.709 & 3.118 & -0.000582 & 0 \\
\hline & $A_{0}^{\prime}$ & 0 & 0.461 & 0.1041 & -0.01753 & -0.2717 \\
\hline & $A_{1}^{\prime}$ & 0 & 0.00968 & -0.417 & -0.395 & 0.843 \\
\hline & $B^{\prime}$ & -0.414 & -0.0606 & 0.2847 & -0.507 & 0 \\
\hline & $C^{\prime}$ & 1.244 & 0.588 & -1.228 & 0.809 & 0 \\
\hline \multirow[t]{4}{*}{$q_{\text {sea }}$} & $A$ & 1.237 & 3.39 & -10.75 & 12.46 & -5.58 \\
\hline & $B_{0}$ & -0.0727 & 0.1748 & -1.392 & 1.711 & -0.796 \\
\hline & $B_{1}$ & 4.29 & 17.87 & -58.1 & 81.9 & -41.4 \\
\hline & $C$ & 14.34 & -44.9 & 119.7 & -158.5 & 75.3 \\
\hline \multicolumn{2}{|c|}{$Q^{2}$} & \multicolumn{5}{|c|}{$100 \mathrm{GeV}^{2} \leq Q^{2} \leq 2500 \mathrm{GeV}^{2}$} \\
\hline & & $s^{0}$ & $s^{1}$ & $s^{2}$ & $s^{3}$ & $s^{4}$ \\
\hline \multirow[t]{5}{*}{$q_{v}$} & $A_{0}$ & 3.058 & 2.474 & 1.002 & 0 & 0 \\
\hline & $A_{1}$ & -2.182 & -4.48 & -0.2259 & 0 & 0 \\
\hline & $A_{2}$ & 1.522 & 4.30 & 1.315 & 0 & 0 \\
\hline & $B$ & 0.517 & 0.0403 & -0.02098 & 0 & 0 \\
\hline & $C$ & 0.1655 & -0.02063 & 0.0537 & 0 & 0 \\
\hline \multirow[t]{7}{*}{$g$} & $A$ & 1.095 & -2.388 & 9.19 & -30.32 & 34.8 \\
\hline & $B$ & -0.441 & -0.907 & 4.68 & -18.66 & 27.17 \\
\hline & $C$ & 10.99 & 4.71 & 28.01 & -127.9 & 164.0 \\
\hline & $A_{0}^{\prime}$ & 0.3010 & 1.275 & -1.563 & 4.10 & -13.37 \\
\hline & $A_{1}^{\prime}$ & -0.1305 & -1.245 & 2.438 & -2.539 & 12.73 \\
\hline & $B^{\prime}$ & -0.489 & 0.955 & -4.40 & 10.22 & -17.13 \\
\hline & $C^{\prime}$ & 1.331 & -0.2481 & 1.950 & -2.072 & 0 \\
\hline \multirow[t]{4}{*}{$q_{\text {sea }}$} & $A$ & 1.287 & -2.069 & 11.57 & -35.7 & 37.4 \\
\hline & $B_{0}$ & -0.2340 & -0.443 & 1.235 & -3.72 & 3.84 \\
\hline & $B_{1}$ & 6.46 & -10.48 & 89.8 & -284.7 & 299.8 \\
\hline & $C$ & 5.35 & 10.11 & -133.7 & 427. & -457. \\
\hline \multirow[t]{6}{*}{$\delta c_{v}$} & $A_{0}$ & 0 & 0.1219 & 6.20 & -25.04 & 30.98 \\
\hline & $A_{1}$ & 0 & 1.913 & -76.9 & 318. & -392. \\
\hline & $A_{2}$ & 0 & -7.16 & 250.3 & -1062. & 1308. \\
\hline & $A_{3}$ & 0 & 3.19 & -230.1 & 1012. & -1250. \\
\hline & $B$ & 0.499 & 3.47 & -15.26 & 19.67 & 0 \\
\hline & $C$ & 0.329 & 8.24 & -38.0 & 46.3 & 0 \\
\hline \multirow[t]{4}{*}{$\delta c_{s e a}$} & $A$ & 0 & -0.02786 & 0.0349 & -0.02223 & 0 \\
\hline & $B_{0}$ & -0.3141 & -0.425 & 0.1564 & 0 & 0 \\
\hline & $B_{1}$ & 4.72 & -5.48 & 2.686 & 0 & 0 \\
\hline & $C$ & 2.961 & 0.776 & -8.28 & 9.78 & 0 \\
\hline
\end{tabular}


TABLE VI. Coefficients of the parametrization for WHIT3 parton distribution in the photon.

\begin{tabular}{|c|c|c|c|c|c|c|}
\hline \multicolumn{2}{|c|}{$Q^{2}$} & \multicolumn{5}{|c|}{$4 \mathrm{GeV}^{2} \leq Q^{2} \leq 100 \mathrm{GeV}^{2}$} \\
\hline & & $s^{0}$ & $s^{1}$ & $s^{2}$ & $s^{3}$ & $s^{4}$ \\
\hline \multirow[t]{5}{*}{$q_{v}$} & $A_{0}$ & 1.882 & 1.213 & 0.697 & 0 & 0 \\
\hline & $A_{1}$ & 0 & -2.361 & -1.136 & 0 & 0 \\
\hline & $A_{2}$ & 0 & 0.528 & 2.406 & 0 & 0 \\
\hline & $B$ & 0.500 & 0.02107 & 0.00413 & 0 & 0 \\
\hline & $C$ & 0.2500 & -0.2376 & 0.2018 & -0.0504 & 0 \\
\hline \multirow[t]{7}{*}{$g$} & $A$ & 8.00 & -28.64 & 55.9 & -57.6 & 23.66 \\
\hline & $B$ & 0 & -0.987 & 0.510 & -0.0667 & 0 \\
\hline & $C$ & 15.00 & 0.331 & 3.50 & 0.892 & 0 \\
\hline & $A_{0}^{\prime}$ & 0 & 0.461 & 0.1041 & -0.01753 & -0.2717 \\
\hline & $A_{1}^{\prime}$ & 0 & 0.00968 & -0.417 & -0.395 & 0.843 \\
\hline & $B^{\prime}$ & -0.414 & -0.0606 & 0.2847 & -0.507 & 0 \\
\hline & $C^{\prime}$ & 1.244 & 0.588 & -1.228 & 0.809 & 0 \\
\hline \multirow[t]{4}{*}{$q_{\text {sea }}$} & $A$ & 1.587 & 5.05 & -11.26 & 7.56 & -1.471 \\
\hline & $B_{0}$ & -0.1006 & 0.2259 & -1.195 & 1.175 & -0.446 \\
\hline & $B_{1}$ & 5.73 & 25.64 & -58.7 & 63.2 & -25.77 \\
\hline & $C$ & 21.36 & -72.9 & 153.2 & -167.9 & 67.4 \\
\hline \multicolumn{2}{|c|}{$Q^{2}$} & \multicolumn{5}{|c|}{$100 \mathrm{GeV}^{2} \leq Q^{2} \leq 2500 \mathrm{GeV}^{2}$} \\
\hline & & $s^{0}$ & $s^{1}$ & $s^{2}$ & $s^{3}$ & $s^{4}$ \\
\hline \multirow[t]{5}{*}{$q_{v}$} & $A_{0}$ & 3.058 & 2.474 & 1.002 & 0 & 0 \\
\hline & $A_{1}$ & -2.182 & -4.48 & -0.2264 & 0 & 0 \\
\hline & $A_{2}$ & 1.522 & 4.30 & 1.315 & 0 & 0 \\
\hline & $B$ & 0.517 & 0.0403 & -0.02097 & 0 & 0 \\
\hline & $C$ & 0.1655 & -0.02064 & 0.0537 & 0 & 0 \\
\hline \multirow[t]{7}{*}{$g$} & $A$ & 1.270 & -2.817 & 5.74 & -13.27 & 12.68 \\
\hline & $B$ & -0.461 & -0.817 & 3.32 & -12.96 & 18.93 \\
\hline & $C$ & 17.21 & 1.257 & 50.5 & -276.1 & 490. \\
\hline & $A_{0}^{\prime}$ & 0.3010 & 1.275 & -1.563 & 4.10 & -13.37 \\
\hline & $A_{1}^{\prime}$ & -0.1305 & -1.245 & 2.438 & -2.539 & 12.73 \\
\hline & $B^{\prime}$ & -0.489 & 0.955 & -4.40 & 10.22 & -17.13 \\
\hline & $C^{\prime}$ & 1.331 & -0.2481 & 1.950 & -2.072 & 0 \\
\hline \multirow[t]{4}{*}{$q_{\text {sea }}$} & $A$ & 1.850 & -3.67 & 27.14 & -106.6 & 130.9 \\
\hline & $B_{0}$ & -0.2299 & -0.497 & 2.464 & -9.95 & 12.32 \\
\hline & $B_{1}$ & 10.42 & -10.74 & 132.7 & -539. & 656. \\
\hline & $C$ & 4.07 & 4.11 & -171.9 & 707. & -859. \\
\hline \multirow[t]{6}{*}{$\delta c_{v}$} & $A_{0}$ & 0 & 0.1219 & 6.20 & -25.04 & 30.98 \\
\hline & $A_{1}$ & 0 & 1.913 & -76.9 & 318. & -392. \\
\hline & $A_{2}$ & 0 & -7.16 & 250.3 & -1062 & 1308. \\
\hline & $A_{3}$ & 0 & 3.19 & -230.1 & 1012. & -1250. \\
\hline & $B$ & 0.499 & 3.47 & -15.26 & 19.67 & 0 \\
\hline & $C$ & 0.329 & 8.24 & -38.0 & 46.3 & 0 \\
\hline \multirow[t]{4}{*}{$\delta c_{\text {sea }}$} & $A$ & 0 & -0.01948 & 0.02861 & -0.02036 & 0 \\
\hline & $B_{0}$ & -0.413 & -0.439 & 0.1810 & 0 & 0 \\
\hline & $B_{1}$ & 5.19 & -7.40 & 3.40 & 0 & 0 \\
\hline & $C$ & 2.359 & 0.977 & -7.73 & 9.48 & 0 \\
\hline
\end{tabular}


TABLE VII. Coefficients of the parametrization for WHIT4 parton distribution in the photon.

\begin{tabular}{|c|c|c|c|c|c|c|}
\hline \multicolumn{2}{|c|}{$Q^{2}$} & \multicolumn{5}{|c|}{$4 \mathrm{GeV}^{2} \leq Q^{2} \leq 100 \mathrm{GeV}^{2}$} \\
\hline & & $s^{0}$ & $s^{1}$ & $s^{2}$ & $s^{3}$ & $s^{4}$ \\
\hline \multirow[t]{5}{*}{$q_{v}$} & $A_{0}$ & 2.540 & 2.000 & 0.718 & 0 & 0 \\
\hline & $A_{1}$ & 0.0623 & -7.01 & 0.1251 & 0 & 0 \\
\hline & $A_{2}$ & -0.1642 & -0.436 & 10.48 & -5.20 & 0 \\
\hline & $B$ & 0.699 & -0.02796 & -0.00365 & 0 & 0 \\
\hline & $C$ & 0.442 & -1.255 & 1.941 & -0.995 & 0 \\
\hline \multirow[t]{7}{*}{$g$} & $A$ & 4.00 & -9.40 & 15.55 & -14.50 & 5.47 \\
\hline & $B$ & 0 & -1.142 & 1.034 & -0.441 & 0 \\
\hline & $C$ & 3.000 & 0.872 & 1.006 & 0.356 & 0 \\
\hline & $A_{0}^{\prime}$ & 0 & 0.602 & 0.509 & -2.054 & 1.392 \\
\hline & $A_{1}^{\prime}$ & 0 & -0.0922 & -1.899 & 4.18 & -2.494 \\
\hline & $B^{\prime}$ & -0.2895 & 0.376 & -1.719 & 1.116 & 0 \\
\hline & $C^{\prime}$ & 1.439 & -0.557 & 0.366 & 0.733 & -0.762 \\
\hline \multirow[t]{4}{*}{$q_{\text {sea }}$} & $A$ & 1.308 & 2.315 & -7.88 & 8.26 & -3.004 \\
\hline & $B_{0}$ & -0.0373 & 0.0563 & -1.133 & 1.185 & -0.418 \\
\hline & $B_{1}$ & 2.103 & 4.85 & -17.81 & 20.62 & -7.94 \\
\hline & $C$ & 7.00 & -10.17 & 26.00 & -29.60 & 12.27 \\
\hline \multicolumn{2}{|c|}{$Q^{2}$} & \multicolumn{5}{|c|}{$100 \mathrm{GeV}^{2} \leq Q^{2} \leq 2500 \mathrm{GeV}^{2}$} \\
\hline & & $s^{0}$ & $s^{1}$ & $s^{2}$ & $s^{3}$ & $s^{4}$ \\
\hline \multirow[t]{5}{*}{$q_{v}$} & $A_{0}$ & 4.27 & 3.096 & 1.619 & 0 & 0 \\
\hline & $A_{1}$ & -4.74 & -6.90 & -2.430 & 0 & 0 \\
\hline & $A_{2}$ & 2.837 & 6.47 & 4.09 & 0 & 0 \\
\hline & $B$ & 0.678 & -0.0394 & 0.01756 & 0 & 0 \\
\hline & $C$ & 0.1728 & -0.02479 & 0.1446 & 0 & 0 \\
\hline \multirow[t]{7}{*}{$g$} & $A$ & 1.384 & -2.455 & 8.94 & -29.06 & 37.1 \\
\hline & $B$ & -0.442 & -0.719 & 2.961 & -12.09 & 19.16 \\
\hline & $C$ & 4.21 & 2.524 & 10.03 & -18.27 & 2.162 \\
\hline & $A_{0}^{\prime}$ & 0.2992 & 1.179 & -1.915 & 7.26 & -18.39 \\
\hline & $A_{1}^{\prime}$ & -0.1600 & -1.114 & 2.939 & -6.66 & 19.23 \\
\hline & $B^{\prime}$ & -0.483 & 0.755 & -3.80 & 10.75 & -19.93 \\
\hline & $C^{\prime}$ & 1.297 & -0.1669 & 1.906 & -2.057 & 0 \\
\hline \multirow[t]{4}{*}{$q_{\text {sea }}$} & $A$ & 1.188 & -1.396 & 8.71 & -25.42 & 24.92 \\
\hline & $B_{0}$ & -0.2448 & -0.419 & 1.007 & -2.689 & 2.517 \\
\hline & $B_{1}$ & 1.942 & -6.04 & 50.3 & -147.8 & 148.1 \\
\hline & $C$ & 5.42 & 6.11 & -53.8 & 163.2 & -171.6 \\
\hline \multirow[t]{6}{*}{$\delta c_{v}$} & $A_{0}$ & 0 & 0.1219 & 6.20 & -25.04 & 30.98 \\
\hline & $A_{1}$ & 0 & 1.913 & -76.9 & 318. & -392 . \\
\hline & $A_{2}$ & 0 & -7.16 & 250.3 & -1062 & 1308. \\
\hline & $A_{3}$ & 0 & 3.19 & -230.1 & 1012. & -1250 . \\
\hline & $B$ & 0.499 & 3.47 & -15.26 & 19.67 & 0 \\
\hline & $C$ & 0.329 & 8.24 & -38.0 & 46.3 & 0 \\
\hline \multirow[t]{4}{*}{$\delta c_{\text {sea }}$} & $A$ & 0 & -0.02821 & -0.0002649 & 0.00704 & 0 \\
\hline & $B_{0}$ & -0.327 & -0.2298 & 0.0350 & 0 & 0 \\
\hline & $B_{1}$ & 1.254 & 0.878 & 0.2086 & 0 & 0 \\
\hline & $C$ & 4.17 & 0.640 & -7.63 & 7.17 & 0 \\
\hline
\end{tabular}


TABLE VIII. Coefficients of the parametrization for WHIT5 parton distribution in the photon.

\begin{tabular}{|c|c|c|c|c|c|c|}
\hline \multicolumn{2}{|c|}{$Q^{2}$} & \multicolumn{5}{|c|}{$4 \mathrm{GeV}^{2} \leq Q^{2} \leq 100 \mathrm{GeV}^{2}$} \\
\hline & & $s^{0}$ & $s^{1}$ & $s^{2}$ & $s^{3}$ & $s^{4}$ \\
\hline \multirow[t]{5}{*}{$q_{v}$} & $A_{0}$ & 2.540 & 2.000 & 0.718 & 0 & 0 \\
\hline & $A_{1}$ & 0.0623 & -7.01 & 0.1251 & 0 & 0 \\
\hline & $A_{2}$ & -0.1642 & -0.436 & 10.48 & -5.20 & 0 \\
\hline & $B$ & 0.699 & -0.02796 & -0.00365 & 0 & 0 \\
\hline & $C$ & 0.442 & -1.255 & 1.941 & -0.995 & 0 \\
\hline \multirow[t]{7}{*}{$g$} & $A$ & 10.00 & -34.0 & 69.0 & -75.3 & 32.3 \\
\hline & $B$ & 0 & -1.126 & 0.926 & -0.393 & 0 \\
\hline & $C$ & 9.00 & 0.481 & 3.20 & -0.347 & 0 \\
\hline & $A_{0}^{\prime}$ & 0 & 0.602 & 0.509 & -2.054 & 1.392 \\
\hline & $A_{1}^{\prime}$ & 0 & -0.0922 & -1.899 & 4.18 & -2.494 \\
\hline & $B^{\prime}$ & -0.2895 & 0.376 & -1.719 & 1.116 & 0 \\
\hline & $C^{\prime}$ & 1.439 & -0.557 & 0.366 & 0.733 & -0.762 \\
\hline \multirow[t]{4}{*}{$q_{\text {sea }}$} & $A$ & 2.227 & 5.72 & -12.95 & 7.22 & -0.2514 \\
\hline & $B_{0}$ & -0.0881 & 0.1465 & -0.975 & 0.782 & -0.2074 \\
\hline & $B_{1}$ & 3.37 & 14.16 & -31.50 & 27.89 & -8.71 \\
\hline & $C$ & 15.81 & -36.3 & 77.1 & -78.1 & 29.48 \\
\hline \multicolumn{2}{|c|}{$Q^{2}$} & \multicolumn{5}{|c|}{$100 \mathrm{GeV}^{2} \leq Q^{2} \leq 2500 \mathrm{GeV}^{2}$} \\
\hline & & $s^{0}$ & $s^{1}$ & $s^{2}$ & $s^{3}$ & $s^{4}$ \\
\hline \multirow[t]{5}{*}{$q_{v}$} & $A_{0}$ & 4.27 & 3.096 & 1.617 & 0 & 0 \\
\hline & $A_{1}$ & -4.74 & -6.90 & -2.417 & 0 & 0 \\
\hline & $A_{2}$ & 2.837 & 6.47 & 4.07 & 0 & 0 \\
\hline & $B$ & 0.678 & -0.0394 & 0.01750 & 0 & 0 \\
\hline & $C$ & 0.1728 & -0.02457 & 0.1440 & 0 & 0 \\
\hline \multirow[t]{7}{*}{$g$} & $A$ & 1.995 & -3.26 & 1.818 & 1.711 & -4.99 \\
\hline & $B$ & -0.466 & -0.610 & 1.691 & -6.68 & 10.19 \\
\hline & $C$ & 10.75 & 5.42 & 6.55 & -22.97 & 18.67 \\
\hline & $A_{0}^{\prime}$ & 0.2992 & 1.179 & -1.915 & 7.26 & -18.39 \\
\hline & $A_{1}^{\prime}$ & -0.1600 & -1.114 & 2.939 & -6.66 & 19.23 \\
\hline & $B^{\prime}$ & -0.483 & 0.755 & -3.80 & 10.75 & -19.93 \\
\hline & $C^{\prime}$ & 1.297 & -0.1669 & 1.906 & -2.057 & 0 \\
\hline \multirow[t]{4}{*}{$q_{\text {sea }}$} & $A$ & 2.318 & -3.76 & 20.26 & -59.5 & 59.0 \\
\hline & $B_{0}$ & -0.2425 & -0.436 & 1.241 & -3.51 & 3.36 \\
\hline & $B_{1}$ & 5.33 & -8.68 & 74.2 & -207.0 & 196.7 \\
\hline & $C$ & 8.48 & 9.31 & -104.1 & 280.1 & -266.3 \\
\hline \multirow[t]{6}{*}{$\delta c_{v}$} & $A_{0}$ & 0 & 0.1219 & 6.20 & -25.04 & 30.98 \\
\hline & $A_{1}$ & 0 & 1.913 & -76.9 & 318. & -392 \\
\hline & $A_{2}$ & 0 & -7.16 & 250.3 & -1062 & 1308. \\
\hline & $A_{3}$ & 0 & 3.19 & -230.1 & 1012. & -1250. \\
\hline & $B$ & 0.499 & 3.47 & -15.26 & 19.67 & 0 \\
\hline & $C$ & 0.329 & 8.24 & -38.0 & 46.3 & 0 \\
\hline \multirow[t]{4}{*}{$\delta c_{\text {sea }}$} & $A$ & 0 & -0.0658 & 0.1059 & -0.0663 & 0 \\
\hline & $B_{0}$ & -0.2750 & -0.476 & 0.1191 & 0 & 0 \\
\hline & $B_{1}$ & 6.37 & -5.32 & 1.986 & 0 & 0 \\
\hline & $C$ & 3.40 & 0.375 & -8.79 & 10.01 & 0 \\
\hline
\end{tabular}


TABLE IX. Coefficients of the parametrization for WHIT6 parton distribution in the photon.

\begin{tabular}{|c|c|c|c|c|c|c|}
\hline \multicolumn{2}{|c|}{$Q^{2}$} & \multicolumn{5}{|c|}{$4 \mathrm{GeV}^{2} \leq Q^{2} \leq 100 \mathrm{GeV}^{2}$} \\
\hline & & $s^{0}$ & $s^{1}$ & $s^{2}$ & $s^{3}$ & $s^{4}$ \\
\hline \multirow[t]{5}{*}{$q_{v}$} & $A_{0}$ & 2.540 & 2.000 & 0.718 & 0 & 0 \\
\hline & $A_{1}$ & 0.0623 & -7.01 & 0.1251 & 0 & 0 \\
\hline & $A_{2}$ & -0.1642 & -0.436 & 10.48 & -5.20 & 0 \\
\hline & $B$ & 0.699 & -0.02796 & -0.00365 & 0 & 0 \\
\hline & $C$ & 0.442 & -1.255 & 1.941 & -0.995 & 0 \\
\hline \multirow[t]{7}{*}{$g$} & $A$ & 16.00 & -61.0 & 127.8 & -139.9 & 59.9 \\
\hline & $B$ & 0 & -1.109 & 0.845 & -0.351 & 0 \\
\hline & $C$ & 15.00 & 0.1596 & 4.18 & -0.1765 & 0 \\
\hline & $A_{0}^{\prime}$ & 0 & 0.602 & 0.509 & -2.054 & 1.392 \\
\hline & $A_{1}^{\prime}$ & 0 & -0.0922 & -1.899 & 4.18 & -2.494 \\
\hline & $B^{\prime}$ & -0.2895 & 0.376 & -1.719 & 1.116 & 0 \\
\hline & $C^{\prime}$ & 1.439 & -0.557 & 0.366 & 0.733 & -0.762 \\
\hline \multirow[t]{4}{*}{$q_{\text {sea }}$} & $A$ & 3.18 & 8.69 & -22.87 & 18.96 & -5.14 \\
\hline & $B_{0}$ & -0.1003 & 0.1603 & -1.037 & 0.944 & -0.2915 \\
\hline & $B_{1}$ & 5.69 & 18.67 & -46.7 & 50.5 & -18.35 \\
\hline & $C$ & 21.49 & -56.5 & 129.3 & -145.9 & 57.5 \\
\hline \multicolumn{2}{|c|}{$Q^{2}$} & \multicolumn{5}{|c|}{$100 \mathrm{GeV}^{2} \leq Q^{2} \leq 2500 \mathrm{GeV}^{2}$} \\
\hline & & $s^{0}$ & $s^{1}$ & $s^{2}$ & $s^{3}$ & $s^{4}$ \\
\hline \multirow[t]{5}{*}{$q_{v}$} & $A_{0}$ & 4.27 & 3.096 & 1.621 & 0 & 0 \\
\hline & $A_{1}$ & -4.74 & -6.90 & -2.439 & 0 & 0 \\
\hline & $A_{2}$ & 2.837 & 6.46 & 4.10 & 0 & 0 \\
\hline & $B$ & 0.678 & -0.0394 & 0.01758 & 0 & 0 \\
\hline & $C$ & 0.1728 & -0.02493 & 0.1451 & 0 & 0 \\
\hline \multirow[t]{7}{*}{$g$} & $A$ & 2.378 & -4.38 & 0.585 & 8.34 & -9.92 \\
\hline & $B$ & -0.479 & -0.607 & 1.458 & -6.03 & 9.33 \\
\hline & $C$ & 17.06 & 4.96 & 24.97 & -158.2 & 295.4 \\
\hline & $A_{0}^{\prime}$ & 0.2992 & 1.179 & -1.915 & 7.26 & -18.39 \\
\hline & $A_{1}^{\prime}$ & -0.1600 & -1.114 & 2.939 & -6.66 & 19.23 \\
\hline & $B^{\prime}$ & -0.483 & 0.755 & -3.80 & 10.75 & -19.93 \\
\hline & $C^{\prime}$ & 1.297 & -0.1669 & 1.906 & -2.057 & 0 \\
\hline \multirow[t]{4}{*}{$q_{\text {sea }}$} & $A$ & 3.34 & -5.61 & 50.0 & -220.7 & 302.8 \\
\hline & $B_{0}$ & -0.2402 & -0.409 & 2.263 & -10.50 & 14.87 \\
\hline & $B_{1}$ & 8.79 & -8.86 & 164.0 & -712 & 973. \\
\hline & $C$ & 9.16 & 9.29 & -278.4 & 1175. & -1592 \\
\hline \multirow[t]{6}{*}{$\delta c_{v}$} & $A_{0}$ & 0 & 0.1219 & 6.20 & -25.04 & 30.98 \\
\hline & $A_{1}$ & 0 & 1.913 & -76.9 & 318. & -392 \\
\hline & $A_{2}$ & 0 & -7.16 & 250.3 & -1062. & 1308. \\
\hline & $A_{3}$ & 0 & 3.19 & -230.1 & 1012. & -1250. \\
\hline & $B$ & 0.499 & 3.47 & -15.26 & 19.67 & 0 \\
\hline & $C$ & 0.329 & 8.24 & -38.0 & 46.3 & 0 \\
\hline \multirow[t]{4}{*}{$\delta c_{\text {sea }}$} & $A$ & 0 & -0.0499 & 0.1026 & -0.0787 & 0 \\
\hline & $B_{0}$ & -0.361 & -0.576 & 0.2257 & 0 & 0 \\
\hline & $B_{1}$ & 7.68 & -8.83 & 3.88 & 0 & 0 \\
\hline & $C$ & 2.548 & 0.691 & -8.70 & 10.65 & 0 \\
\hline
\end{tabular}




\section{FIGURES}

FIG. 1. The data and the theoretical predictions for the photon structure function $F_{2}^{\gamma} / \alpha$. The vertical axes show $F_{2}^{\gamma} / \alpha$ and the horizontal axes represent the scaling variable $x$. The data points with a cross mark are not used for the fit, according to the selection criterion Eq. (3.1). The OPAL data points are obtained by removing the direct charm quark contribution. We hence drop from the theoretical curves the $\gamma^{*} \gamma \rightarrow c \bar{c}$ contributions. The remaining structure function data contain all hadronic final states. (a) The best fits with WHIT1-WHIT3 gluon distributions. (b) The best fits with WHIT4-WHIT6 gluon distributions.

FIG. 2. The deviation of each data point from the best fit, $F_{2}^{\gamma}\left(x, Q^{2}\right)_{\text {fit }}-F_{2}^{\gamma}\left(x, Q^{2}\right)_{\text {data }}$ divided by the error $\sigma\left(F_{2}^{\gamma}\left(x, Q^{2}\right)\right.$ data $)$. A data point with smaller $x$ value is placed lower in each data set with a common $\left\langle Q^{2}\right\rangle$. The data points with a simple cross mark are removed from the fit by the criterion Eq. (3.1).

FIG. 3. The $C_{g}$ dependence of the total $\chi^{2}$ for fixed $A_{g}$ 's when the standard valence-quark distributions are taken for $A_{g}=0.5$ (solid line) and 1.0 (dashed line). The large square, diamond and cross marks are the minimal $\chi^{2}$ values as obtained by tuning the valence-quark distributions.

FIG. 4. Gluon distributions at $Q^{2}=4,20$ and $100 \mathrm{GeV}^{2}$. The top 3 figures are for WHIT1 (solid), WHIT2 (dotted) and WHIT3 (dashed), the middle 3 figures are WHIT4 (solid), WHIT5 (dotted) and WHIT6 (dashed), and the bottom 3 figures are GRV [9](solid), DG [4] (dotted) and LAC1 [10](dashed) for comparison.

FIG. 5. Predictions for the charm quark distribution in the photon, calculated by the QPM (solid lines) and by the massive inhomogeneous AP equations (dash-dotted lines). The valence-up-quark distributions of WHIT1-WHIT3 (dashed lines) and WHIT4-WHIT6 (dotted lines) are also shown for comparison. 
FIG. 6. The differential cross section of the process $e^{+} e^{-} \rightarrow e^{+} e^{-} c \bar{c}$ at TRISTAN energy $(\sqrt{s}=58 \mathrm{GeV})$, evaluated exactly and via EPA: (a) near the charm quark pair threshold $\left(\sqrt{\hat{s}}=m_{c c}=4 \mathrm{GeV}\right)$ and; (b) at far above the threshold $\left(\sqrt{\hat{s}}=m_{c \bar{c}}=15 \mathrm{GeV}\right)$. Vertical bars indicate errors of numerical integration of the exact matrix elements. We set $m_{c}=1.5 \mathrm{GeV}$ and $\alpha=1 / 137$.

FIG. 7. The invariant mass distribution of charm quark pair in the process $e^{+} e^{-} \rightarrow e^{+} e^{-} c \bar{c}$ evaluated exactly and via EPA at $\sqrt{s}=58 \mathrm{GeV}$. We set $m_{c}=1.5 \mathrm{GeV}$, and $\alpha=1 / 137$

FIG. 8. The leading order prediction for the total cross section of the inclusive process $e^{+} e^{-} \rightarrow e^{+} e^{-} c \bar{c} X$. The contributions from the resolved photon processes depend on the parton distributions WHIT1 to 6 . The curves are obtained by setting $m_{c}=1.5 \mathrm{GeV}, \alpha=1 / 137$ and $\Lambda_{4}=0.4 \mathrm{GeV}$ for the strong coupling, and by requiring the invariant mass $W$ of the hadron system to satisfy $W \geq 2 m_{D}=3.74 \mathrm{GeV}$. The vertical bars attached to the WHIT1, WHIT4 and WHIT6 predictions indicate the dependence of the cross sections on the charm mass $m_{c}$ between $1.3 \mathrm{GeV}$ and $1.7 \mathrm{GeV}$.

FIG. 9. The leading order predictions for the total cross sections of the inclusive process $e^{+} e^{-} \rightarrow e^{+} e^{-} c \bar{c} X$, where contributions of the direct and resolved photon processes are shown separately. The parton distributions of WHIT1 (a) and WHIT6 (b) are used to calculate the resolved photon contributions. We set $m_{c}=1.5 \mathrm{GeV}, \alpha=1 / 137$ and $\Lambda_{4}=0.4 \mathrm{GeV}$. The vertical bars indicate the dependence of the cross sections on the charm mass $m_{c}$ between $1.3 \mathrm{GeV}$ and 1.7 $\mathrm{GeV}$. 
Fig. 1 (a)
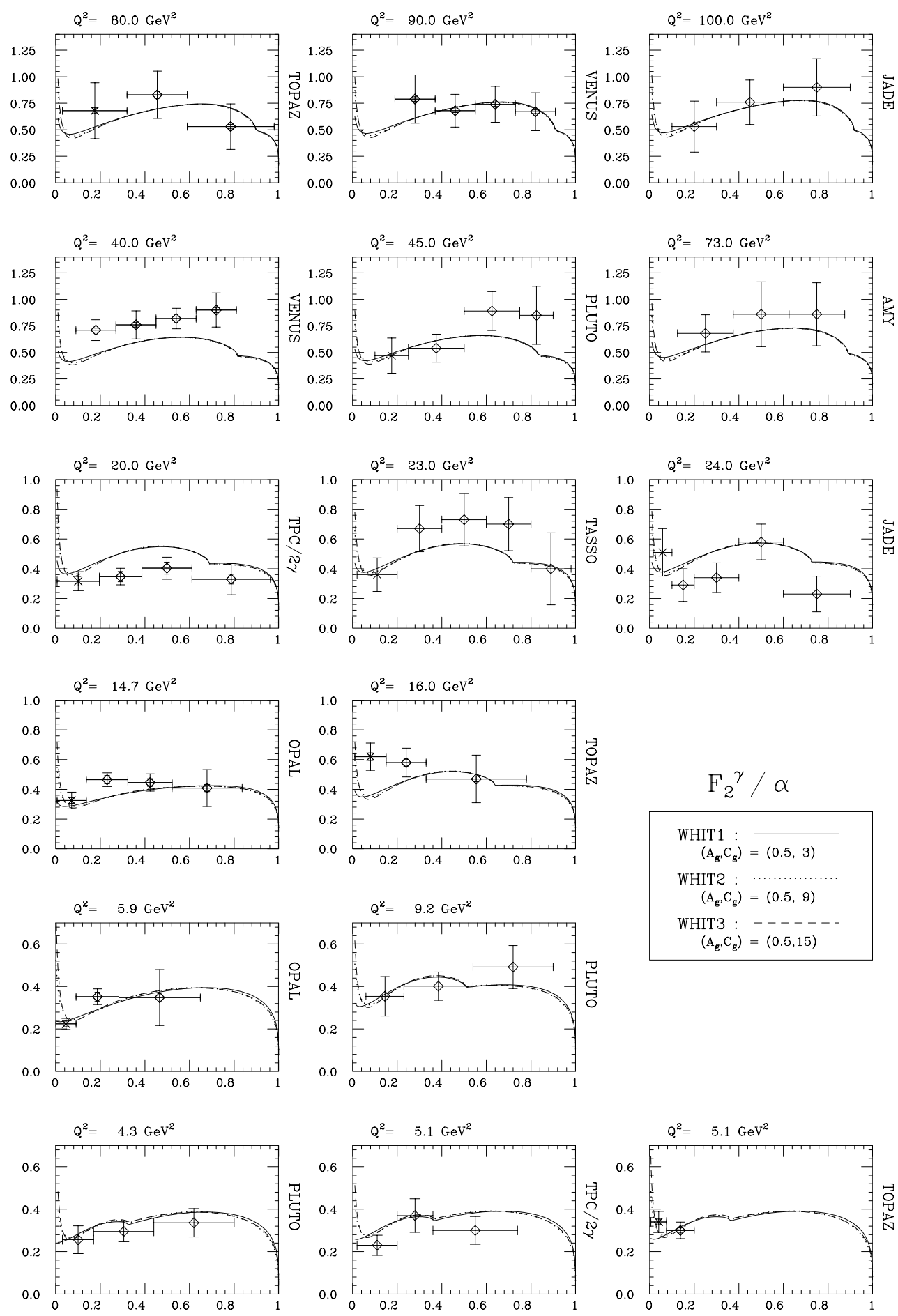
Fig. 1 (b)
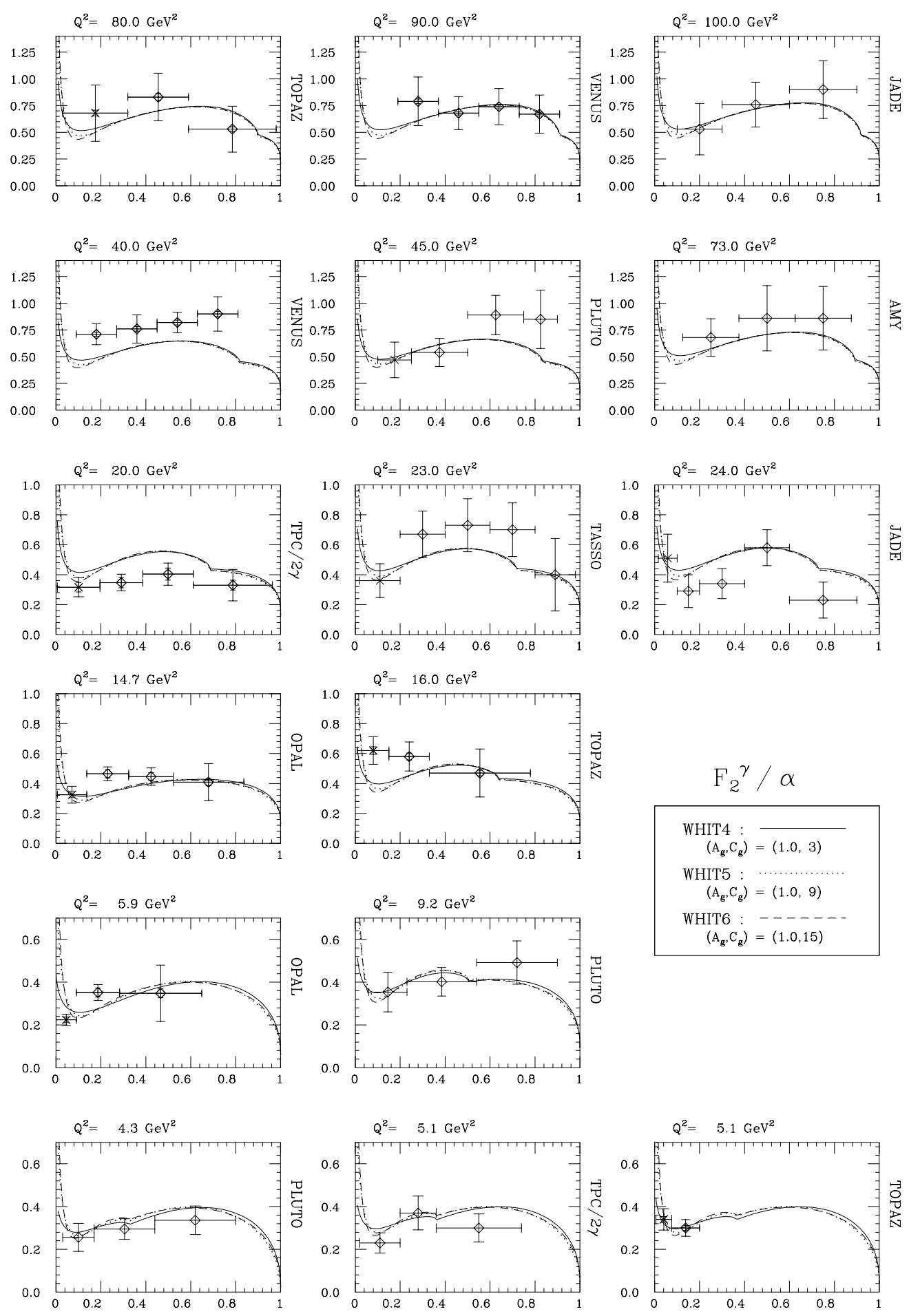
Fig. 2
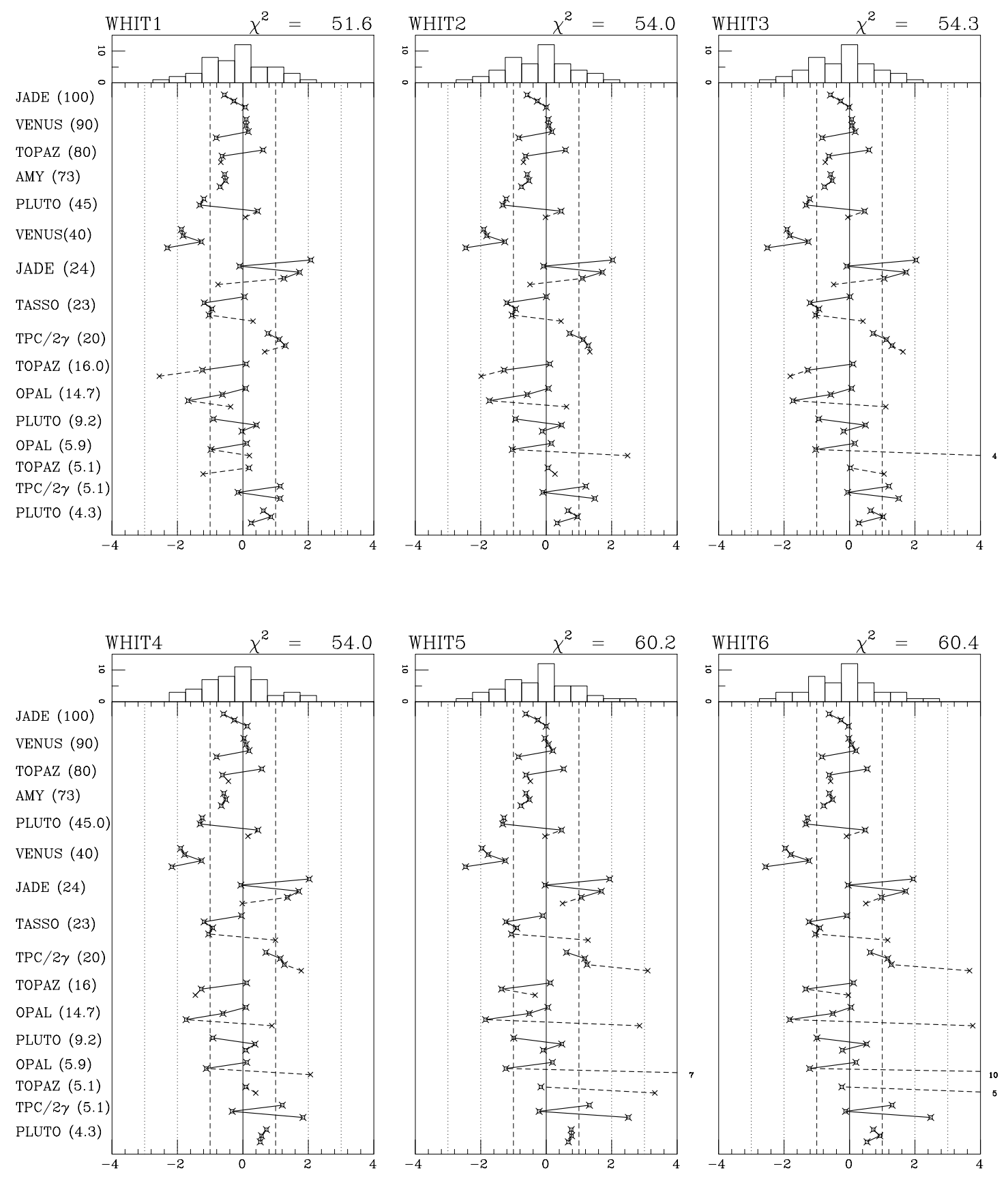


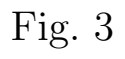

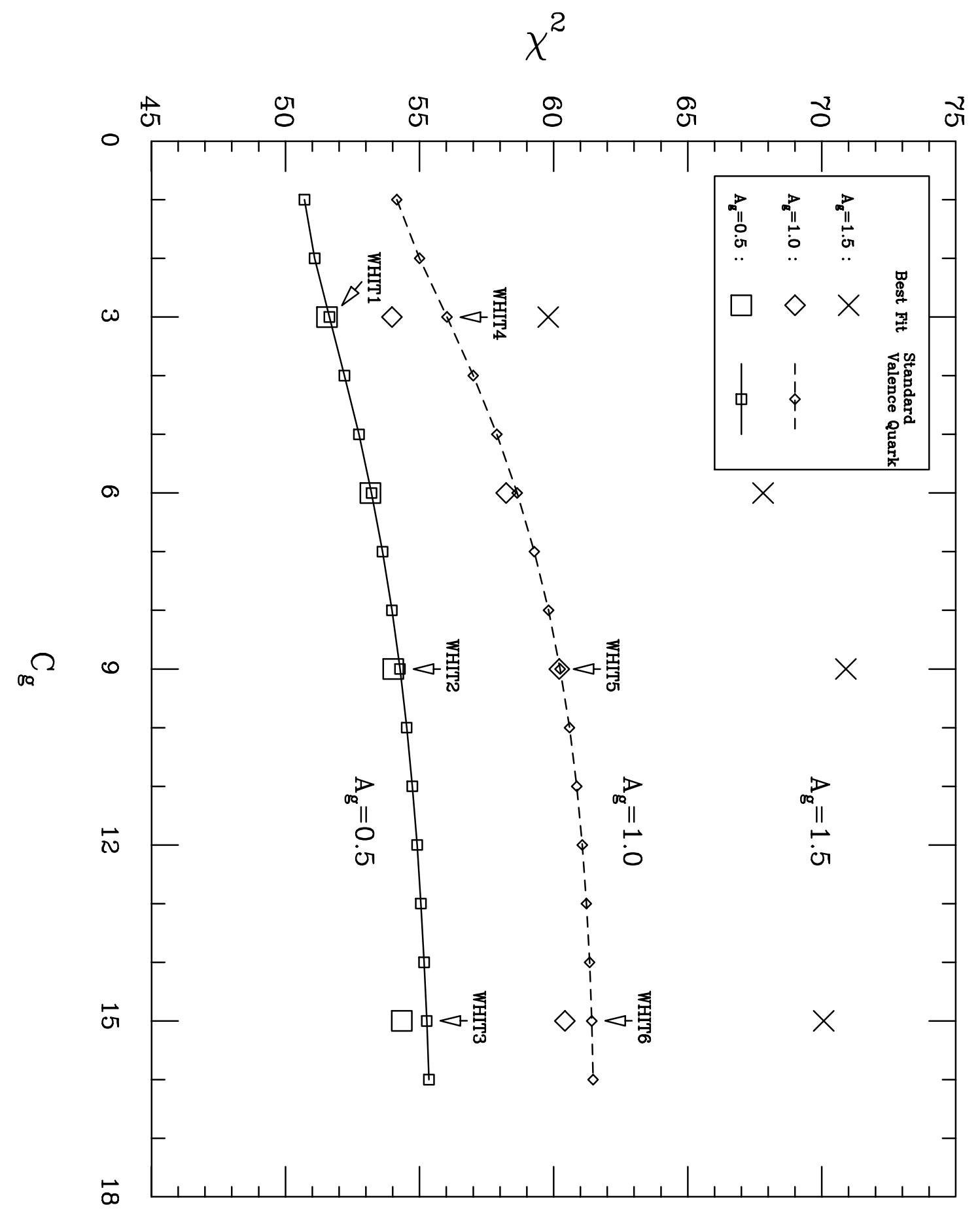


Fig. 田

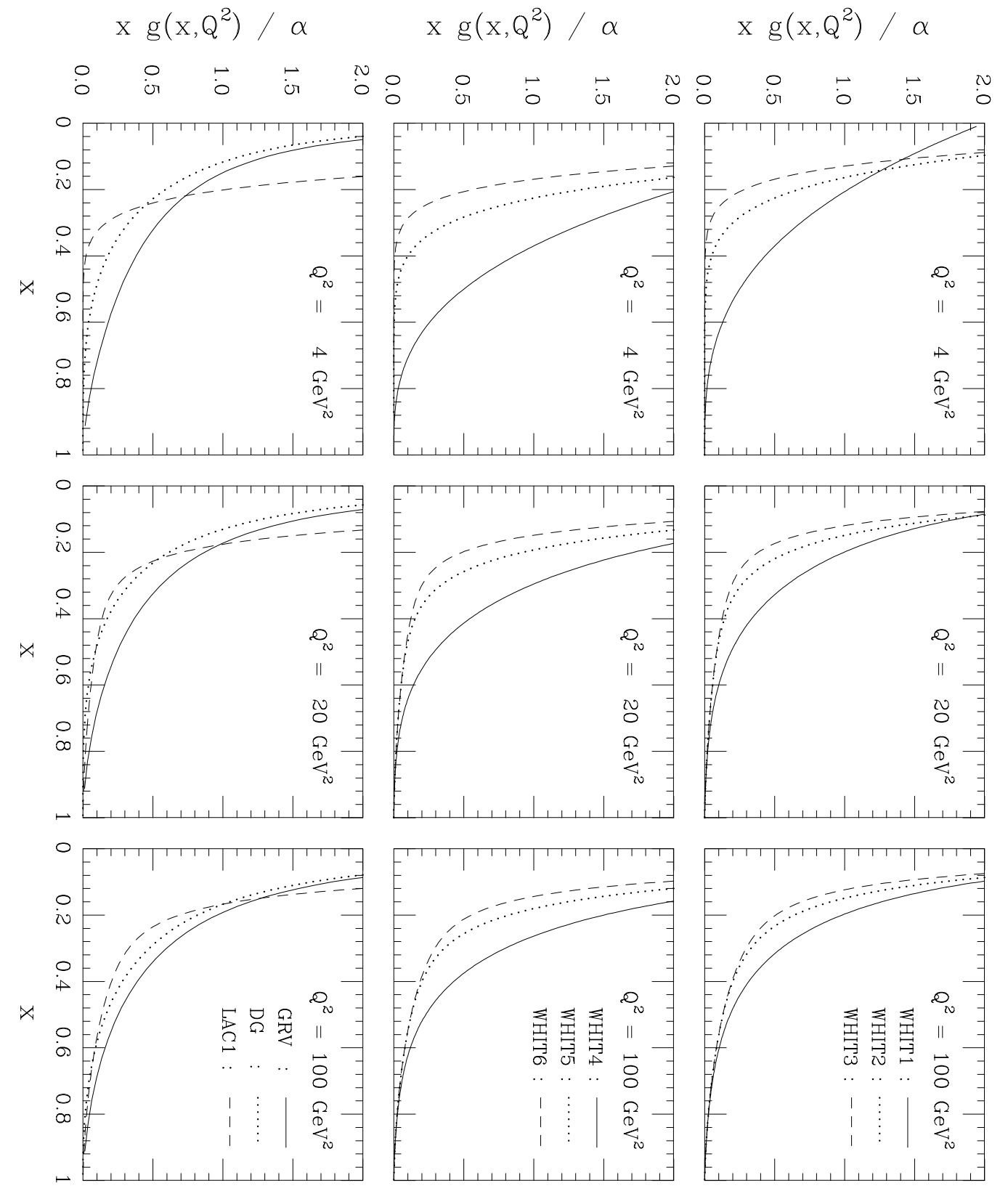


Fig. 5
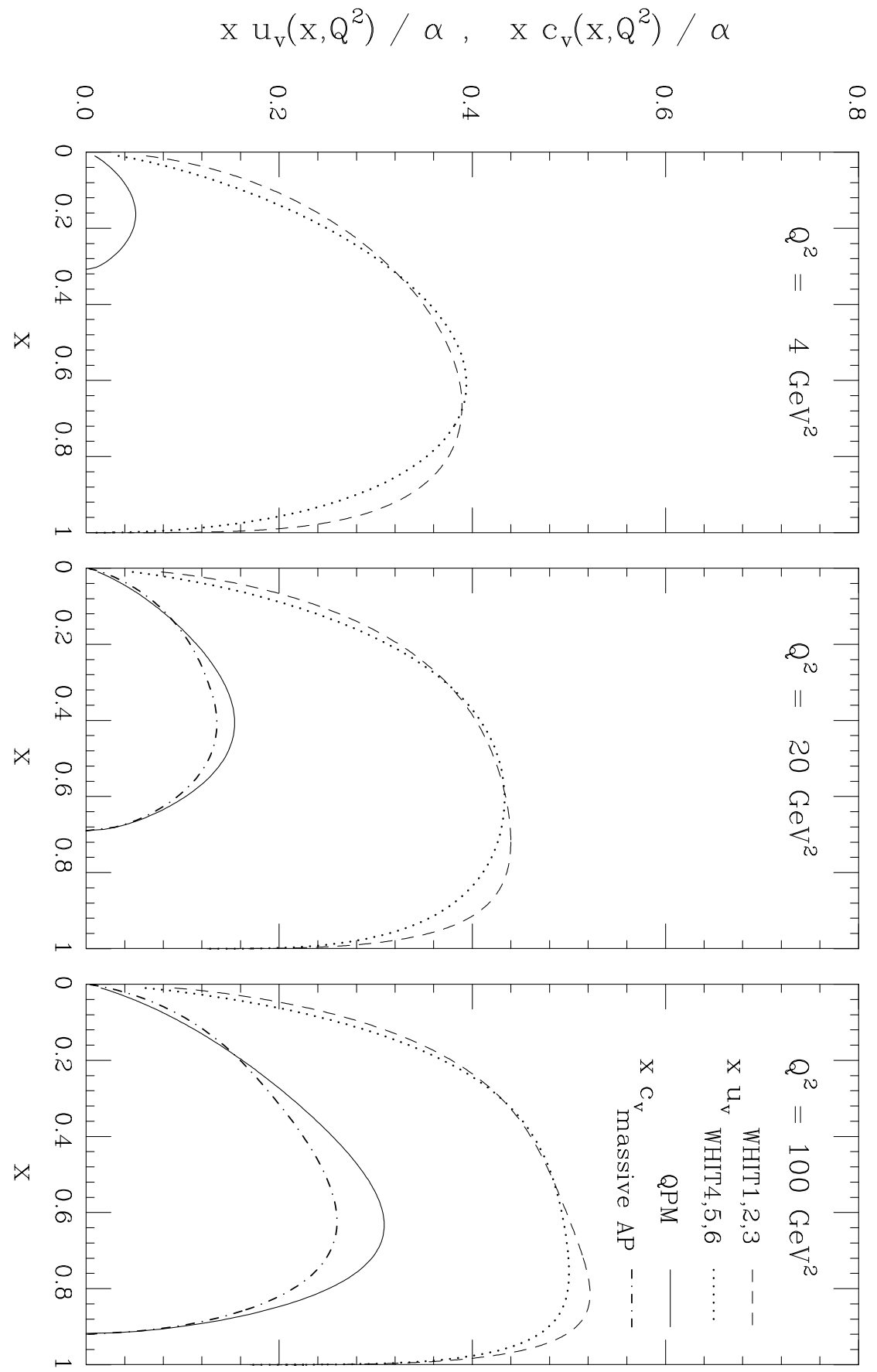
Fig. [6 (a)

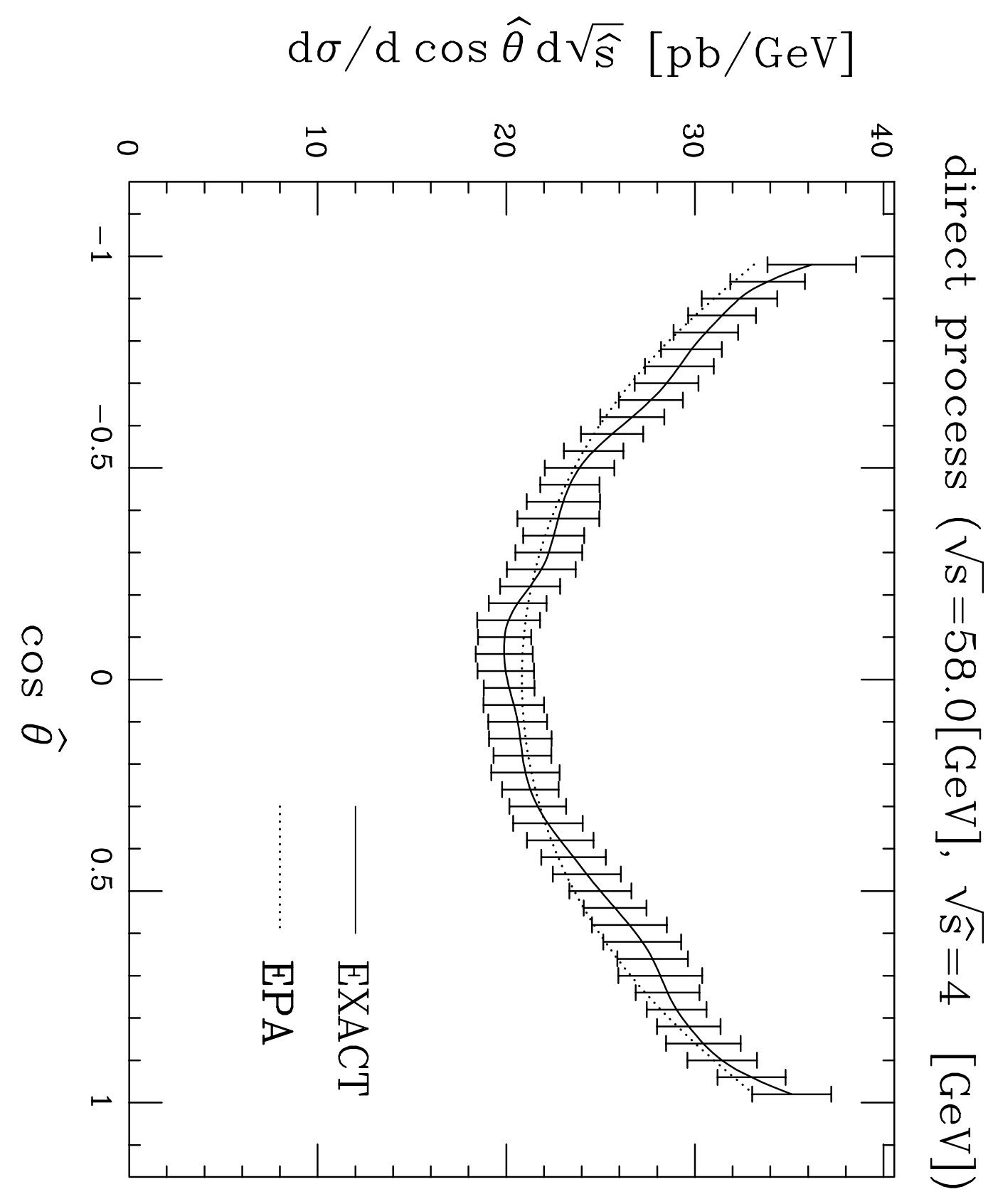


Fig. 6 (b)

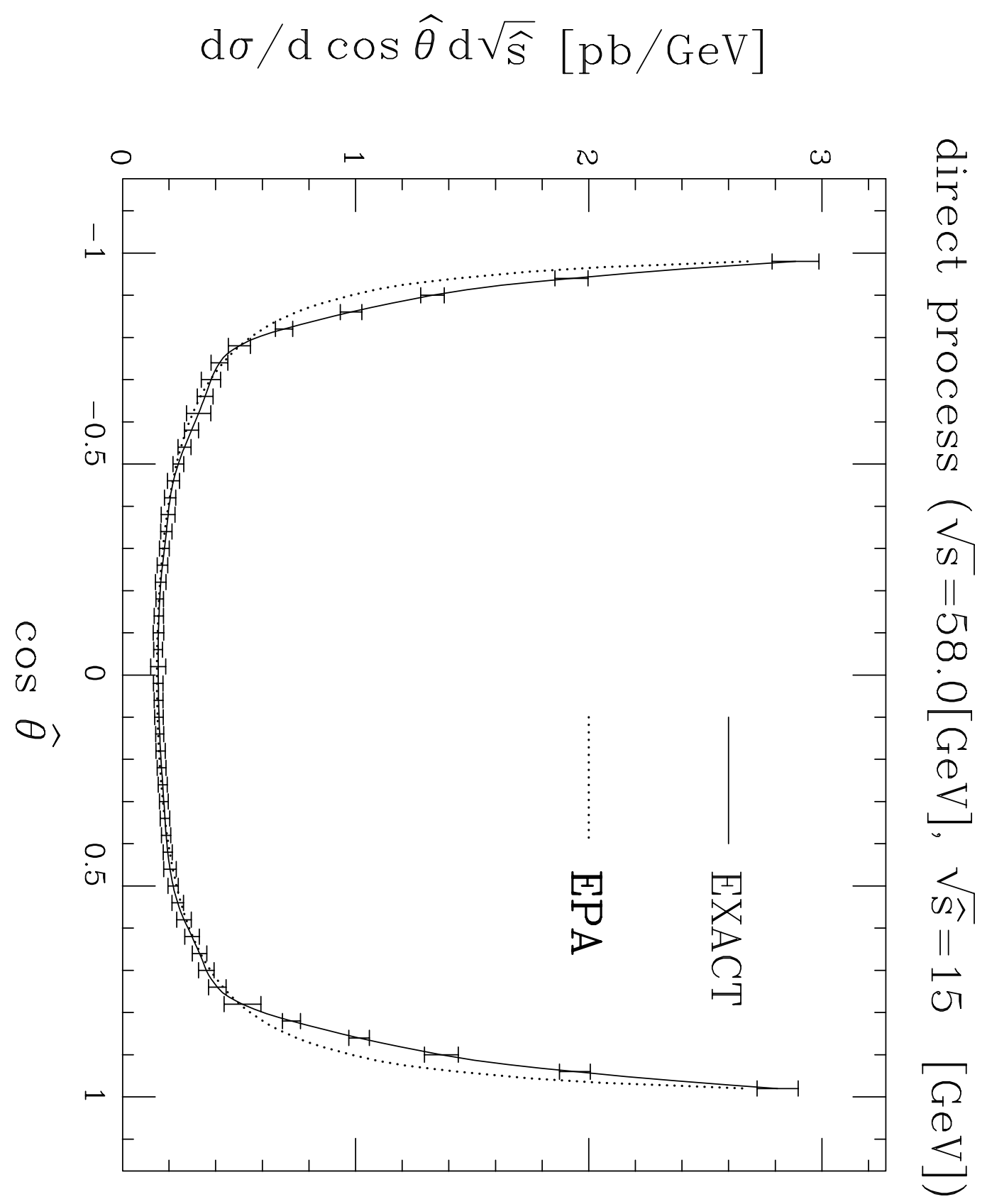


Fig. 7

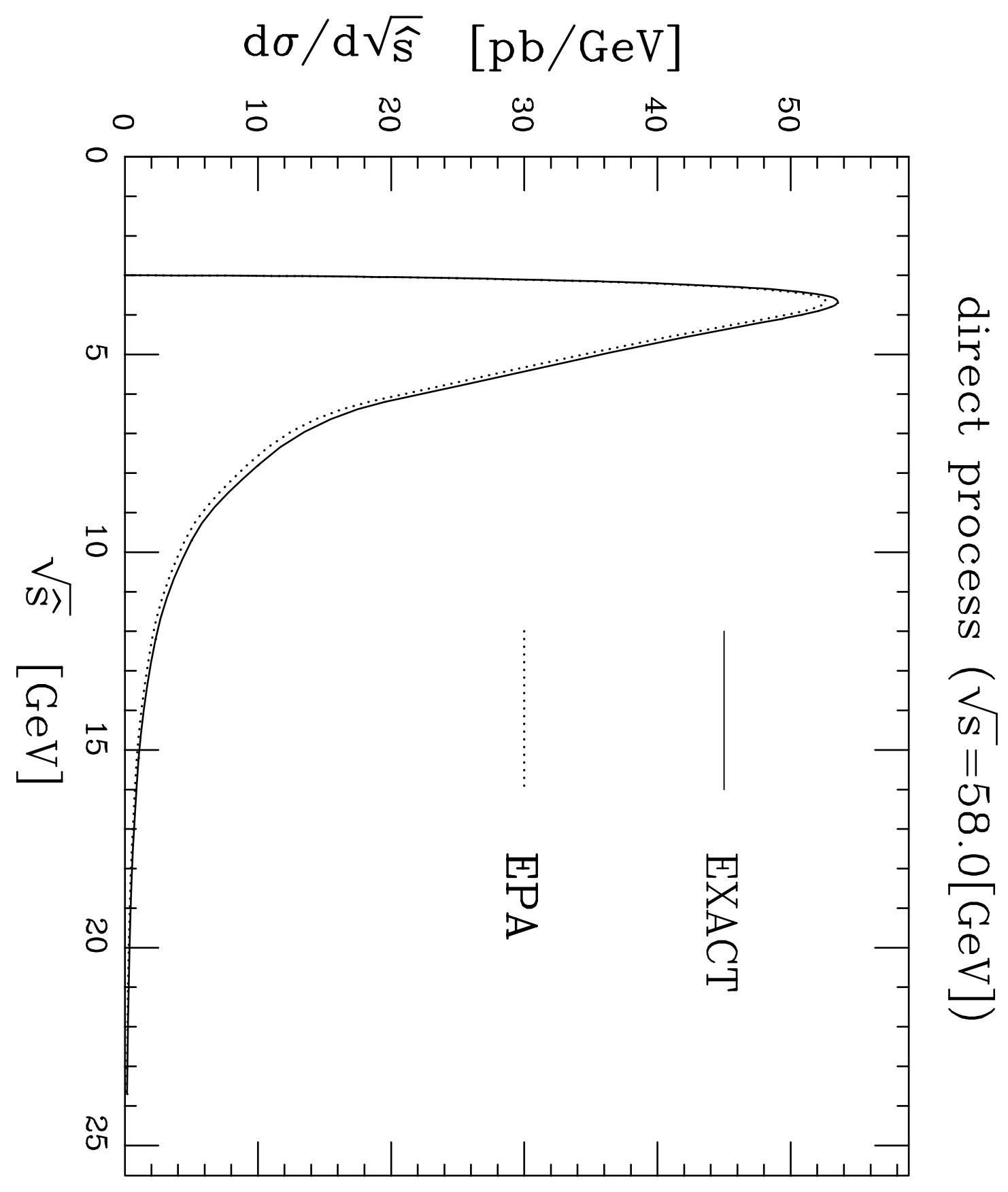


Fig. 8

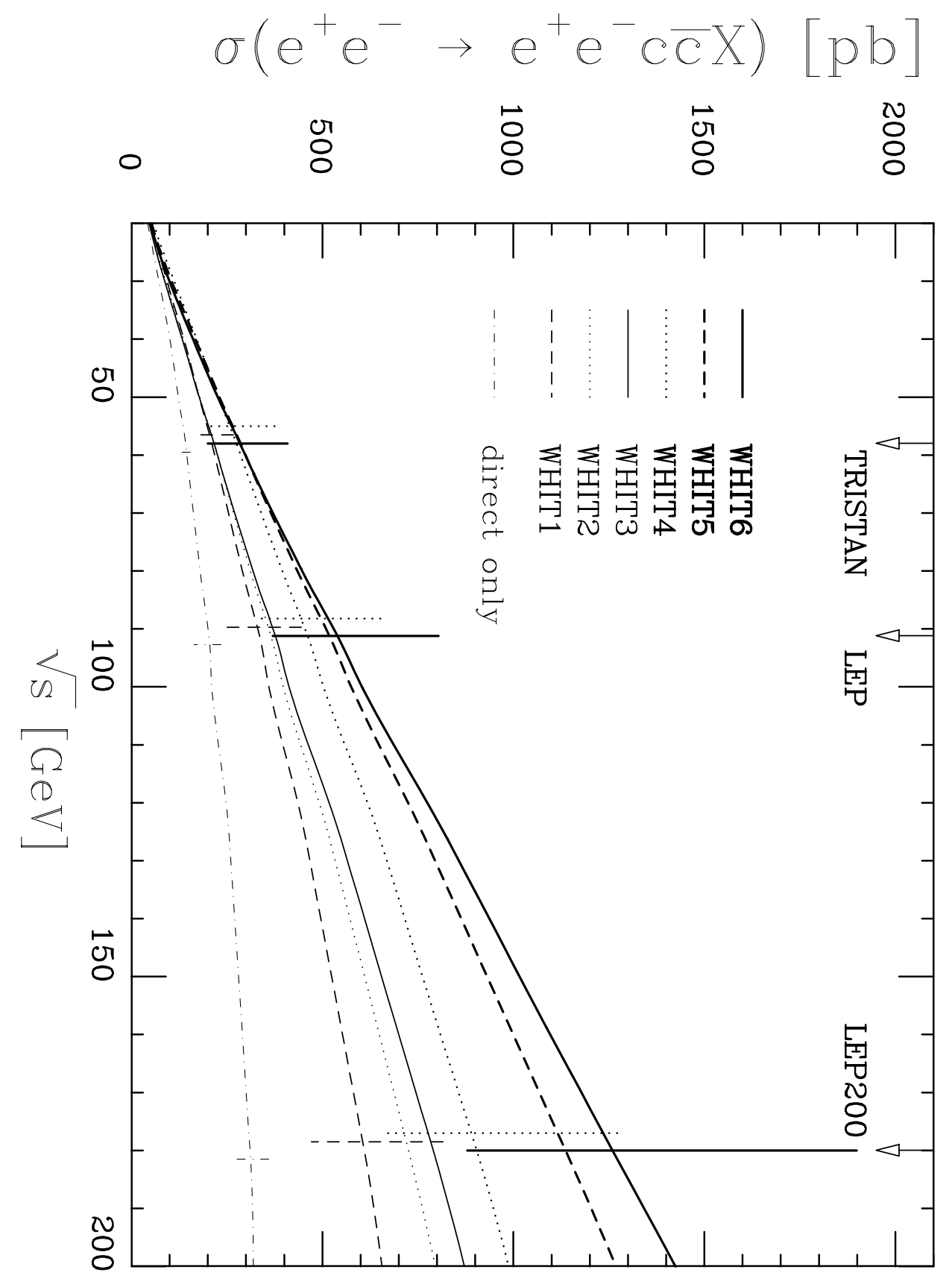


Fig. 9 (a)

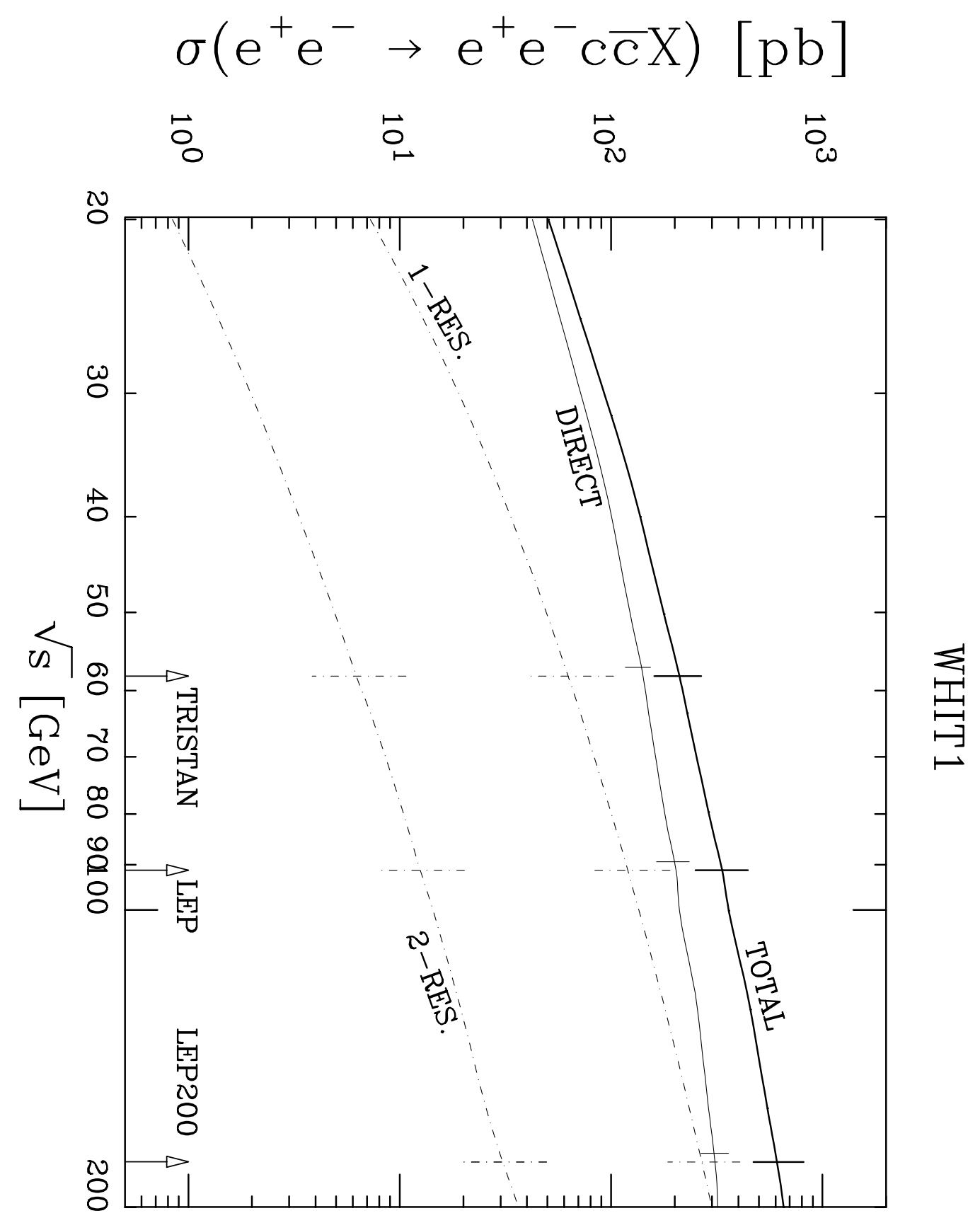


Fig. 9 (b)

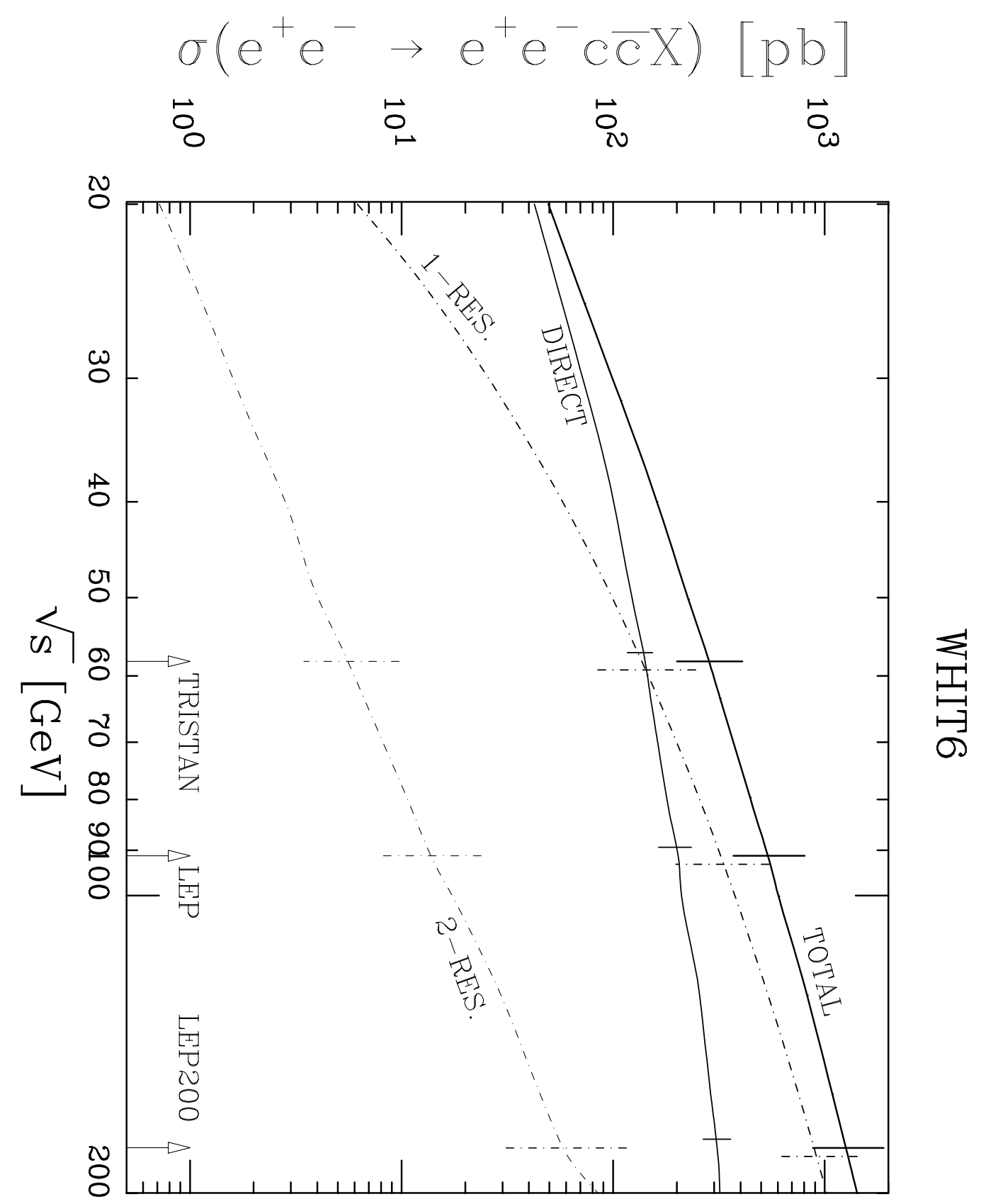

